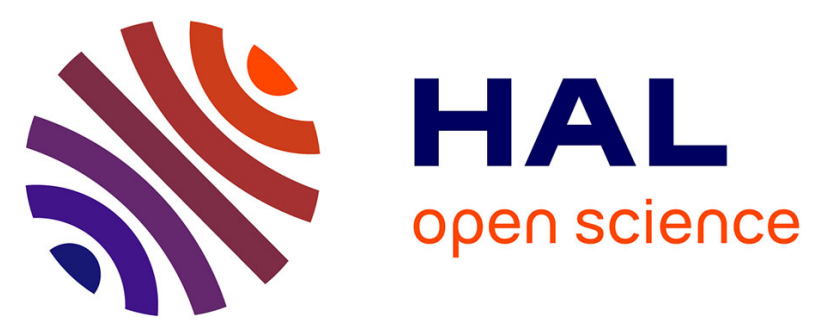

\title{
New state estimators and communication protocol for distributed event-triggered consensus of linear multi-agent systems with bounded perturbations
}

Christophe Viel, Sylvain Bertrand, Michel Kieffer, Helene Piet-Lahanier

\section{To cite this version:}

Christophe Viel, Sylvain Bertrand, Michel Kieffer, Helene Piet-Lahanier. New state estimators and communication protocol for distributed event-triggered consensus of linear multi-agent systems with bounded perturbations. IET Control Theory and Applications, 2017, 11, pp.1736 - 1748. 10.1049/ietcta.2016.0856 . hal-01576589

HAL Id: hal-01576589

https://hal-centralesupelec.archives-ouvertes.fr/hal-01576589

Submitted on 23 Aug 2017

HAL is a multi-disciplinary open access archive for the deposit and dissemination of scientific research documents, whether they are published or not. The documents may come from teaching and research institutions in France or abroad, or from public or private research centers.
L'archive ouverte pluridisciplinaire HAL, est destinée au dépôt et à la diffusion de documents scientifiques de niveau recherche, publiés ou non, émanant des établissements d'enseignement et de recherche français ou étrangers, des laboratoires publics ou privés. 


\title{
New state estimators and communication protocol for distributed event-triggered consensus of linear multi-agent systems with bounded perturbations
}

\author{
Christophe Viel*, Sylvain Bertrand*, Michel Kieffer**, Helene Piet-Lahanier*
}

*ONERA - The French Aerospace Lab, F-91123 Palaiseau, France (e-mail: firstname.lastname@onera.fr). ** L2S, Univ Paris-Sud, CNRS, CentraleSupelec, 91190 Gif-sur-Yvette, France

(e-mail: firstname.lastname@lss.supelec.fr)

$16 / 01 / 2017$

Abstract - This paper extends recent results by Garcia $e$ t al. on event-triggered communication to reach consensus in multi-agent systems. First, it studies the effect of two types of additive and bounded state perturbations on the consensus and on the communications. Second, it describes an improved agent state estimator as well as an estimator of the state estimation uncertainty to trigger communications. Convergence to consensus is studied. Simulations show the effectiveness of the proposed estimators in presence of state perturbations.

Index Terms - consensus, distributed event-triggered control, multi-agent system.

\section{Introduction}

Consensus is an important problem in cooperative control, see [2, 7, 8, 15, 19]. In such problem, several agents have to be synchronized to the same state. For example, $[10,17]$ study application of consensus to solve the problem of time synchronization in wireless sensor networks (WSN), when the communication delay is negligible. When the control is distributed, consensus usually requires significant exchange of information between neighbouring agents so that each agent can properly evaluate its control law.

Communication may either be permanent [15, 19], with continuous-time exchange of information, or performed at discrete time instants, which is more practical. In the latter case, agents may broadcast information at a constant period $T_{c}$ [7], usually slotted to avoid message collisions. This method requires a good clock synchronization among agents. Alternatively, communications may be triggered only when necessary, to save communication energy.

With distributed control based on event-triggered communications, each agent estimates the state of its neighbours to evaluate its control law [9]. Each agent also estimates its own state with the information available to its neighbours. As soon as the error between this estimate and its actual state reaches some threshold, a communication is triggered. In [5], the agent dynamic is a single integrator and the considered threshold decreases with time while reaching the consensus. This implies an increase of the frequency of communications. In [18], the dynamic is a double integrator and the communication triggering condition (CTC) depends on a state-independent threshold exponentially decreasing with time. The communication frequency reduces compared to [5] but still increases close to consensus. General linear dynamics are considered in $[6,8,20]$. State-dependent thresholds are then considered to ensure some convergence property for the system. These previous approaches were developed for noise-free dynamics and prove sensitive to perturbations. This issue has been partly addressed by $[3,12]$ who propose an event-triggered method to mitigate the impact of perturbations in the case of dynamics described by simple integrators. 
This paper addresses the problem of distributed event-triggered communications for consensus of a multi-agent system with both general linear dynamics and state perturbations. This work extends results presented in [6,8] by analyzing the effect of state perturbations on the consensus and on the communications. Moreover, to reduce communications, this paper proposes an improved estimator of the agent states, derives an estimator of the estimation error, and introduces an adapted communication protocol. By taking into account the control input of the agents, the proposed estimator allows to obtain a consensus with much less communications than with the approach in $[6,8]$. The proposed technique is thus well-suited to applications where communications should be minimized, e.g., to improve furtivity, reduce energy consumption, or limit collisions between transmitted data packets. Application examples with such constraints are exposed in $[13,14]$ for the case of a fleet of vehicles, or in [1] where agents aim at merging local feature-based maps.

With this approach, estimates of the states of all the agents (not only neighboring ones) are required to evaluate all control laws. More estimates are performed, but this reduces the communication frequency. A convergence analysis is achieved while considering state perturbations composed of two components: one common to all agents, and one agent-specific.

After introducing some notations in Section 2, the problem is formulated in Section 3. The CTC, presented in Section 4, requires a new state estimator, described in Section 5, along with a communication protocol. A second estimator is exposed in Section 5.4 to obtain an implementable distributed event-triggering strategy presented in Section 6. Section 7 compares the performance of the proposed approach to state-of-the-art results from $[6,8]$. The main notations are provided in Table 1.

\begin{tabular}{|c|l|}
\hline$x_{i}$ & state of Agent $i$. \\
\hline \hline$y_{i}^{j}$ & estimate of the state $x_{i}$ performed by Agent $j$. \\
\hline$y^{j}$ & vector gathering the estimates performed by Agent $j$ of the states of all agents. \\
\hline$y$ & vector $\left[\left(y_{1}^{1}\right)^{T} \quad \ldots \quad\left(y_{N}^{N}\right)^{T}\right]^{T}$ of estimates performed by each agent of its own state \\
\hline$v^{i}$ & common estimate of $y^{i}$ performed by all agents. \\
\hline$e_{i}^{j}$ & estimation error between $x_{i}$ and $y_{i}^{j}$. \\
\hline$t_{j, k}$ & transmission time of the $k$-th message sent by Agent $j$. \\
\hline$t_{j, k}^{i}$ & reception time by Agent $i$ of the $k$-th message sent by Agent $j$. \\
\hline$t_{\ell}^{i}$ & reception time by Agent $i$ of the $\ell$-th message, whatever the sending agent. \\
\hline
\end{tabular}

Table 1 Main notations

\section{Preliminaries}

Classical notations introduced in what follows are taken from [4].

Consider a network of $N$ agents which topology is described by an undirected graph $\mathcal{G}=(\mathcal{N}, \mathcal{E})$, where $\mathcal{N}=$ $\{1,2, \ldots, N\}$ is the set of nodes and $\mathcal{E} \subset \mathcal{N} \times \mathcal{N}$ the set of edges. The set of neighbours of an Agent $i$ is $\mathcal{N}_{i}=$ $\{j \in \mathcal{N} \mid(i, j) \in \mathcal{E}, i \neq j\} . N_{i}$ is the cardinal number of $\mathcal{N}_{i}$. The weighted adjacency matrix of $\mathcal{G}$ at time $t$ is $\mathrm{A}=\left[a_{i j}\right]_{N \times N}$ with non-negative elements $a_{i j}$. The graph is balanced or undirected iff $\forall(i, j) a_{i j}=a_{j i}$, else it is directed. An edge $\epsilon_{i j} \in \mathcal{E}$ if and only if its weight $a_{i j}=1$ and represents an ordered pair of nodes $(i, j)$, where $i$ and $j$ are respectively the parent and child nodes. A graph is connected when there is a path linking any pair of vertices. When $a_{i j}(t)=1$, Node $i$ is able to send information to Node $j$ at time $t$. If this is not possible, $a_{i j}(t)=0$.

Let $1_{N}=[1,1, \ldots, 1]^{T} \in \mathbb{R}^{N \times 1}$ be the all-one vector and $I_{N} \in \mathbb{R}^{N \times N}$ be the identity matrix of size $N$. The Laplacian matrix is $L$. $L$ is symmetric iff $\mathcal{G}$ is undirected. In all cases, $L$ satisfies $L 1_{N}=0$ and has only one null eigenvalue $\lambda_{1}(L)$ and all its non-zeros eigenvalues $\lambda_{2}(L) \leq \lambda_{3}(L) \leq \ldots \leq \lambda_{N}(L)$ are strictly positive. The smallest eigenvalue, the smallest strictly positive eigenvalue, and the maximum eigenvalue of a matrix $M$ are respectively denoted $\lambda_{\min }(M), \lambda_{\min >0}(M)$, and $\lambda_{\max }(M)$. 
Finally, the Kronecker product is denoted as $\otimes$.

\section{Problem statement}

As in [8], the communication graph is assumed to be fixed, undirected, and connected. Moreover, the dynamic equations of an Agent $i$ are

$$
\begin{aligned}
& \dot{x}_{i}(t)=A x_{i}(t)+B u_{i}(t)+d_{i}(t) \\
& u_{i}(t)=c_{1} F \sum_{j \in \mathcal{N}_{i}}\left(y_{i}^{i}(t)-y_{j}^{i}(t)\right) .
\end{aligned}
$$

In (1), $x_{i} \in \mathbb{R}^{n}$ is the state of Agent $i, u_{i} \in \mathbb{R}^{m}$ is its control input evaluated using $y_{j}^{i} \in \mathbb{R}^{n}$, the estimate of the state of Agent $j$ performed by Agent $i$ as described in Section 5. $A \in \mathbb{R}^{n \times n}$ and $B \in \mathbb{R}^{n \times m}$. One has $c_{1}=c+c_{2}$ with $c=1 / \lambda_{2}(L)$ and $c_{2} \geq 0$ a design parameter. $F=-B^{T} P$ where $P$ is a symmetric positive semi-definite matrix, solution of the Riccati equation

$$
P A+A^{T} P-2 P B B^{T} P+2 \alpha P<0
$$

with $\alpha>0$.

Remark 1. It can be noted than the parameter $c$ is related to the Laplacian matrix $L$, which requires the knowledge of the communication graph $\mathcal{G}$ by each agent to evaluate its control input (2). Since $\mathcal{G}$ is fixed, it can be assumed that the communication graph is initially known by all agents, or that a flooding method like exposed in Section 5.3 can be intiated at $t=0$ to deduce it. Thus, $L$ and $c$ can be computed by each agent and the control input $u_{i}$ can be evaluated in a fully distributed way.

Contrary to [8], one considers here additive perturbation $d_{i} \in \mathbb{R}^{n}$. This perturbation is assumed to be such that

$$
d_{i}(t)=m(t)+s_{i}(t)
$$

where $m(t) \in \mathbb{R}^{n}$ is a bounded time-varying perturbation with $\|m(t)\| \leq M_{\max }$ identical for all agents and $s_{i}(t) \in$ $\mathbb{R}^{n}$ is a bounded agent-specific perturbation with for all $i=1, \ldots, N\left\|s_{i}(t)\right\| \leq S_{\max }$, where $M_{\max } \geq 0$ and $S_{\max } \geq 0$ are known bounds. This two-parts additive perturbation model can be used, e.g., to represent the combined effect of a uniform wind field on a fleet of aircrafts and specific attitude-dependent turbulences affecting differently each aircraft.

The vector of all state perturbations is then

$$
d(t)=1_{N} \otimes m(t)+s(t)
$$

with $s(t)=\left[s_{1}(t)^{T} \ldots s_{N}(t)^{T}\right]^{T}$.

The problem considered here consists in designing a distributed control scheme, robust to perturbations, to drive the agents to a bounded consensus, while limiting the communications between agents. For that purpose, communication time instants are chosen locally by Agent $i$ using an event-triggered approach introduced in Section 4.

Definition 1. The network of agents reaches a bounded consensus iff there exists some $\varepsilon>0$ such that

$$
\lim _{t \rightarrow \infty}\left\|x_{i}(t)-x_{j}(t)\right\| \leqslant \varepsilon
$$

for all pairs of agents $(i, j) \in \mathcal{E}$.

In this paper, as in [8], we suppose that there is no communication delay and agents know perfectly their own state. 


\section{Event-triggered consensus}

This section introduces an event-triggered strategy to reduce the number of communications in Theorem 1 . For that purpose, we assume that the estimates $y_{i}^{j}$ for all $i$ and $j$, are perfectly known by all agents in the network. This imposes strong constraints on the estimators embedded in each agent and on the communication protocol. These constraints will be relaxed in Section 5.3 to allow a practical implementation of the proposed technique.

Let $\widehat{L}=L \otimes P, \bar{L}=\widehat{L} A_{c}+A_{c}^{T} \widehat{L}, A_{c}=\bar{A}+\bar{B}_{1}, \bar{A}=I_{N} \otimes A, \bar{B}_{1}=c_{1} L \otimes(B F), M=P B B^{T} P$, and

$$
\beta=\frac{\lambda_{\min >0}(-\bar{L})}{\lambda_{\max }(\widehat{L})} .
$$

It is proven in [8] that $\bar{L}$ is semi-definite negative. In the following theorem, the initial states are considered to be known by all agents.

Theorem 1. Assume that $(A, B)$ is controllable and that the communication graph is connected and undirected with a fixed topology described by the Laplacian matrix $L$. Consider some design parameter $\eta>0$. Agents with dynamics (1) achieve a bounded consensus with

$$
\forall(i, j) \quad \lim _{t \rightarrow \infty}\left\|x_{i}-x_{j}\right\|^{2} \leq \frac{N^{3} \eta}{\beta \lambda_{\min }(P)}
$$

if the bound on the agent-specific perturbation satisfies

$$
S_{\max } \leq \sqrt{\frac{\alpha\left\|c_{2} \lambda_{2}(L) M\right\|}{\lambda_{\max }(P)}} \sqrt{\frac{N \eta}{\lambda_{\min }(P) \beta}}
$$

and if communications are triggered when

$$
\bar{\delta}_{i} \geq \rho z_{i}^{T} \Theta z_{i}+\eta
$$

with $\Theta_{i}=\left(2 c_{2}-b_{i} N_{i}\left(c_{2}-c\right)\right) M, 1 \geq \rho>0$, and

$$
\begin{aligned}
\bar{\delta}_{i}= & c_{1}\left[\left(z_{i}-N_{i} e_{i}^{i}\right)^{T} M \sum_{j \in \mathcal{N}_{i}} \Delta_{i j}+\frac{N_{i}}{2 b_{i}} e_{i}^{i T} M e_{i}^{i}\right. \\
& \left.+\left(1+\frac{b_{i}}{2}\right) N_{i} \sum_{j \in \mathcal{N}_{i}}\left(\Delta_{i j}^{T} M \Delta_{i j}\right)\right] \\
& +2\left(c_{2}-c\right) N_{i} z_{i}^{T} M e_{i}^{i}+\left[2 c\left(N_{i}\right)^{2}\left(1+b_{i}\right)+\frac{c_{2}-c}{b_{i}} N_{i}\right. \\
& \left.+c N_{i}(N-1)\left(b_{i}+\frac{3}{b_{i}}\right)\right] e_{i}^{i T} M e_{i}^{i}
\end{aligned}
$$

where $z_{i}=\sum_{j \in \mathcal{N}_{i}}\left(y_{i}^{i}-y_{j}^{i}\right), e_{i}^{i}=y_{i}^{i}-x_{i}, \Delta_{i j}=y_{i}^{j}-y_{i}^{i}, 0<b_{i}<\frac{2 c_{2}}{\left(c-c_{2}\right) N_{i}}$ if $c_{2}>c, b_{i}>0$ otherwise.

The proof of Theorem 1 is in Appendix A.1.

From (8) and (10), one sees that $\eta$ can be used to adjust the trade-off between the bound on the consensus error and the amount of triggered communications. If $\eta=0$ and if there is no perturbation, the system achieves an asymptotic consensus.

The CTC (10) mainly depends on $e_{i}^{i}$ and $\Delta_{i j}$. A communication is triggered by Agent $i$ when the estimate $y_{i}^{i}$ of its own state $x_{i}$ is not satisfying, i.e., when $e_{i}^{i}$ becomes large. It is also triggered when the discrepancy $\Delta_{i j}$ between this estimate and that made by other agents $y_{i}^{j}$ with their available information is large. 
The two perturbations have a direct impact on $e_{i}^{i}$ and thus on the frequency of communications. The sufficient condition (9) on $S_{\max }$ to have a consensus depends on $\eta$ and on the measure of connectivity $\lambda_{2}(L)$ of the graph. Systems with more connected graphs are more robust to perturbations. $M_{\max }$ does neither influence the quality of the consensus, nor its convergence.

To reduce the number of communications triggered, one has to keep $\bar{\delta}_{i}$ as small as possible. This is done by keeping $e_{i}^{i}$ and $\Delta_{i j}$ small, which is achieved by building accurate estimates $y_{i}^{i}$ and $y_{i}^{j}$, as described in Section 5. Then, since in a distributed context, the $y_{i}^{j}$ s cannot be easily made available to all agents, the CTC introduced in Theorem 1 is difficult to implement. This issue is addressed in Section 5.4.

\section{Agents state estimation and communication protocol}

In what follows, the time instant at which the $k$-th message has been sent by Agent $j$ is denoted $t_{j, k}$. Let $t_{j, k}^{i}$ be the time at which the $k$-th message sent by Agent $j$ has been received by Agent $i$. Since in this paper, one has assumed that there is no communication delay between agents, $t_{j, k}^{i}=t_{j, k}$ for all $i \in \mathcal{N}_{j}$. The time of reception by Agent $i$ of the $\ell$-th message is $t_{\ell}^{i}$, whatever the sending agent.

\subsection{Agents state estimation}

In [8], the estimate $y_{j}^{i}(t)$ is evaluated as

$$
\begin{aligned}
y_{j}^{i}\left(t_{j, k}^{i}\right) & =x_{j}\left(t_{j, k}^{i}\right), \\
\dot{y}_{j}^{i}(t) & =A y_{j}^{i}(t), \quad t_{j, k}^{i} \leq t<t_{j, k+1}^{i} .
\end{aligned}
$$

Let $y^{i}=\left[y_{1}^{i T}, y_{2}^{i T}, \ldots y_{N}^{i T}\right]^{T} \in \mathbb{R}^{N n}$ be the vector gathering the estimates of the states of all agents performed by Agent $i$. The vector $y^{T}=\left[\begin{array}{lll}\left(y_{1}^{1}\right)^{T} & \ldots & \left(y_{N}^{N}\right)^{T}\end{array}\right] \in \mathbb{R}^{N n}$ gathers the estimates performed by each agent of its own state. Similarly, let $e^{T}=\left[\begin{array}{lll}\left(e_{1}^{1}\right)^{T} & \ldots & \left(e_{N}^{N}\right)^{T}\end{array}\right] \in \mathbb{R}^{N n}$.

Here, the estimate $y_{j}^{i}(t)$ is evaluated as

$$
\begin{aligned}
\dot{y}_{j}^{i}(t) & =A y_{j}^{i}(t)+B \tilde{u}_{j}^{i}(t), \quad t_{j, k}^{i} \leq t<t_{j, k+1}^{i} \\
\tilde{u}_{j}^{i}(t) & =c_{1} F \sum_{p \in \mathcal{N}_{j}}\left(y_{j}^{i}(t)-y_{p}^{i}(t)\right) \\
y_{j}^{i}\left(t_{j, k}^{i}\right) & =x_{j}\left(t_{j, k}^{i}\right),
\end{aligned}
$$

where (14) takes into account the control input of the agents. Considering all the agents, (14)-(16) can be rewritten as

$$
\begin{aligned}
\dot{y}^{i}(t) & =A_{c} y^{i}(t) \\
y_{j}^{i}\left(t_{j, k}^{i}\right) & =x_{j}\left(t_{j, k}^{i}\right),
\end{aligned}
$$

where $A_{c}=\bar{A}+\bar{B}_{1}, \bar{A}=I_{N} \otimes A$, and $\bar{B}_{1}=c_{1} L \otimes(B F)$.

To determine the control inputs applied by Agent $j$, Agent $i$ needs to perform an estimate of the state of all the neighbours of Agent $j$. As the communication graph is connected, Agent $i$ will have to evaluate the state of all agents in the network to determine the control inputs applied by all other agents.

Remark 2. If there is no perturbation, i.e., $M_{\max }=0$ and $S_{\max }=0$, the estimate error $e_{i}^{i}$ vanishes. Moreover, in absence of perturbation, if for some time instant $t_{k}, y^{i}\left(t_{k}\right)=y^{j}\left(t_{k}\right)$ for all $(i, j) \in \mathcal{N}$, then $y^{i}(t)=y^{j}(t)$ for all $(i, j)$ for all $t>t_{k}$. As a consequence, $\Delta_{i j}(t)=0$ and $e_{i}^{i}(t)=0$ for all $(i, j)$ for all $t>t_{k}$. No communication will be triggered for $t>t_{k}$. 


\subsection{Communication protocol: fully-connected graph}

In this section, the communication graph is assumed as fully connected. As in [8], the message broadcast by an Agent $i$ at $t_{i, k}$ contains the state $x_{i}\left(t_{i, k}\right)$ of Agent $i$. Agent $j \in \mathcal{N}_{i}=\mathcal{N}$ uses it to update its estimate $y_{i}^{j}$ according to (16).

With a fully connected graph, the information transmitted by some agent is received without delay by all other agents in the network. As a consequence, one has $y_{i}^{i}(t)=y_{i}^{j}(t)$ and $\Delta_{i j}=0$ for all $(i, j) \in \mathcal{N}^{2}$.

In this case, the CTC in Theorem 1 can be evaluated. Communications are triggered mainly due to the state perturbations.

\subsection{Communication protocol: not fully-connected graph}

From now, the communication graph is no more fully connected. Assume first that a message broadcast by Agent $i$ at $t_{i, k}$ contains only its state $x_{i}\left(t_{i, k}\right)$. Only neighbouring agents receive the message and use $x_{i}\left(t_{i, k}\right)$ to update their estimates $y_{i}^{j}, j \in \mathcal{N}_{i}$, according to (16).

A relaying is necessary to allow other agents updating $y_{i}^{j}, j \notin \mathcal{N}_{i}$. Two strategies are discussed in what follows.

5.3.1. Flooding Method: With the first strategy, a message received by an agent is immediately retransmitted to its neighbours.

When an Agent broadcasts a message at $t_{i, k}$, this message contains $t_{i, k}$ and the state $x_{i}\left(t_{i, k}\right)$ of Agent $i$. When some Agent $j$, neighbour of Agent $i$, receives this message, it broadcasts $t_{i, k}$ and $x_{i}\left(t_{i, k}\right)$ to its own neighbours if it has not done it previously. This message is further broadcast by the neighbours. This is a typical flooding strategy [11, 16], which enables all the network receiving the message.

Since there is no communication delay, one has $y_{i}^{i}(t)=y_{i}^{j}(t)$ and $\Delta_{i j}=0$ for all $(i, j) \in \mathcal{N}^{2}$ as in Section 5.2.

With this method, each time a communication is triggered for a given agent, the same message is broadcast up to $N$ times, depending on the topology. This technique is not competitive compared to that presented in [8].

5.3.2. Delayed flooding method: With the proposed alternative strategy, when a message is received by some agent, this agent waits until its CTC is satisfied to broadcast its own state as well as updated estimates of the states of all agents in the network evaluated from information in the messages received from its neighbours. This requires to store and broadcast a vector containing the time instants at which the communication has been triggered for each agent.

Thus, when a communication is triggered at $t_{i, k}$, Agent $i$ first updates $y_{i}^{i}\left(t_{i, k}\right)=x_{i}\left(t_{i, k}\right)$. Then, instead of transmitting only $t_{i, k}$ and $x_{i}\left(t_{i, k}\right)$, it broadcasts the vector $y^{i}$ and a vector

$$
T^{i}=\left[t_{1, k_{1}}, \ldots, t_{i-1, k_{i-1}}, t_{i, k}, t_{i+1, k_{i+1}} \ldots t_{N, k_{N}}\right]
$$

of time instants, where each $t_{j, k_{j}}$ represents the time at which the triggering condition of Agent $j$ has been satisfied. When some Agent $\ell$ receives the message from Agent $i$, it compares the time instants in $T^{i}$ with those of its own $T^{\ell}$. Each components of $y^{\ell}$ such that $t_{i, k}>t_{\ell, k}$, i.e., corresponding to a more recent triggering instant, are replaced by those of $y^{i}$. The vector $T^{\ell}$ is updated accordingly.

Example 1 illustrates this information diffusion strategy.

Example 1. In Figure 1(a), the CTC is satisfied at $t_{1,1}$ for Agent 1. It updates its own estimate $y_{1}^{1}=x_{1}$ and the first component of $T^{1}$ with $t_{1,1}$. Then it broadcasts $T^{1}$ and $y^{1}$. Its neighbours, Agents 2 and 5 , receive this message. Agent 2, since the first component $t_{1,1}$ of $T^{1}$ is more recent than that of $T^{2}$, updates $y_{1}^{2}$ as $y_{1}^{2}=y_{1}^{1}$. The first component of $T^{2}$ is now $t_{1,1}$. Agent 5 performs the same updates. 
In Figure 1(b), the CTC is satisfied for Agent 2 which performed the update $y_{2}^{2}=x_{2}$ and sets the second component of $T^{2}$ to $t_{2,1}$. It broadcasts then $T^{2}$ and $y^{2}$. Agent 3 , once it receives this message, using $T^{2}$, knows that its estimates of the states of Agents 1 and 2 are outdated and performs the updates $y_{1}^{3}=y_{1}^{2}$ and $y_{2}^{3}=y_{2}^{2}$. The two first components of $T^{3}$ are now $t_{1,1}$ and $t_{2,1}$. Agent 1 updates only $y_{2}^{1}=y_{2}^{2}$ and the second component of $T^{1}$ to $t_{2,1}$. A similar behavior is observed in Figure 1(c).In Figure 1(d), the CTC is satisfied simultaneously for Agents 1 and 4. Since the first components of $T^{1}$ is larger than that of $T^{4}$,i.e., $t_{1,2}>t_{1,1}$, Agent 5 uses $y_{1}^{1}$ coming from Agent 1 to update $y_{1}^{5}$. It uses $y_{4}^{4}$ coming from Agent 4 to update $y_{4}^{5}$.

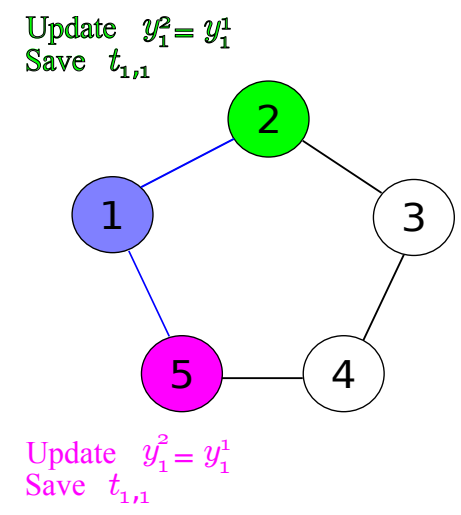

(a) CTC satisfied for Agent 1.

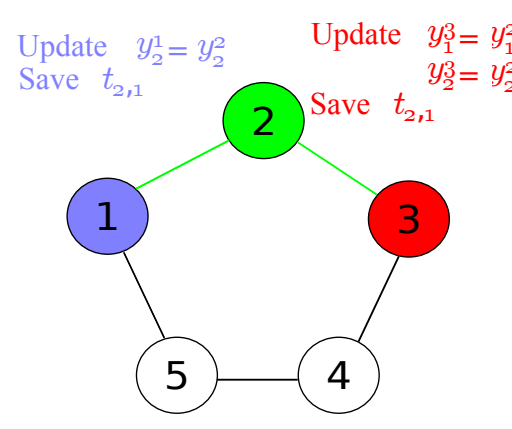

(b) CTC satisfied for Agent 2.

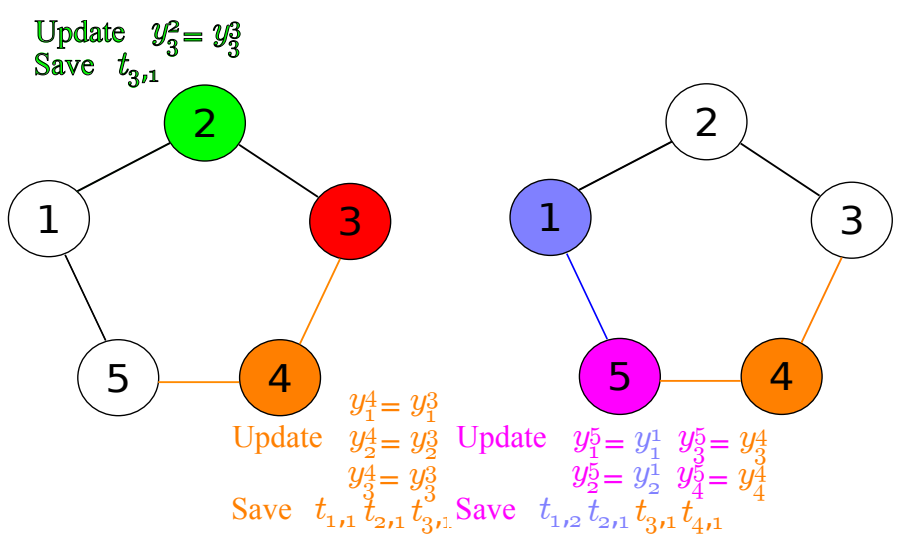

(c) CTC satisfied for Agent 3. (d) CTC satisfied simultaneously for Agents 1 and 4.

Figure 1: Example : Communication relay

The proposed communication protocol has been designed so that once a message has been sent, $(i)$ the estimation error $e_{i}^{i}$ and discrepancies $\Delta_{i j}$ are reset to zero, and (ii) the CTC in Theorem 1, is no longer satisfied.

\subsection{Estimation $\boldsymbol{v}^{i}$ of estimate $\boldsymbol{y}^{i}$ by Agent $j$}

The delayed flooding protocol of Section 5.3.2 allows each Agent $i$ having access to $y_{j}^{i}$, for all $j \in \mathcal{N}$. Nevertheless, Agent $i$ is not able to access $y_{i}^{j}$, which is required to evaluate its CTC in Theorem 1. To address this issue, each Agent $i$ evaluates an additional estimates $v^{j}=\left[v_{1}^{j T} \ldots v_{N}^{j T}\right]^{T} \in \mathbb{R}^{N n}$ of $y^{j}$ for all $j \in \mathcal{N}_{i} \cup\{i\}$, with the constraint that the estimates $v^{i}$ performed by Agents $i$ and $j \in \mathcal{N}_{i}$ have to be identical. For that purpose, the estimate $v^{i}$ performed by Agent $i$ and all its neighbours $j \in \mathcal{N}_{i}$ is updated only when the CTC is satisfied for Agent $i$ and when it broadcasts a message. The $v^{j}$ s are thus less frequently updated than the $y^{i}$ s and are less accurate. Both estimators are evaluated simultaneously by each agent. Introducing $v^{j}$ does not require any modification of the delayed flooding protocol. Agent $i$ uses the $v^{i}$ s to check the CTC and $y^{i}$ to evaluate the control inputs.

The dynamics of the additional estimate $v^{i}$ is

$$
\begin{aligned}
\dot{v}_{j}^{i}(t) & =A v_{j}^{i}(t)+B \bar{u}_{j}^{i}(t), \quad t_{k}^{i} \leq t<t_{k+1}^{i} \\
\bar{u}_{j}^{i}(t) & =c_{1} F \sum_{p \in \mathcal{N}_{\ell}}\left(v_{j}^{i}(t)-v_{p}^{i}(t)\right) \\
v^{i}\left(t_{i, k}\right) & =y^{i}\left(t_{i, k}\right) \\
v_{j}^{i}\left(t_{j, k}\right) & =y_{j}^{j}\left(t_{j, k}\right), \quad j \in \mathcal{N}_{i} .
\end{aligned}
$$


Considering all the agents, (19)-(22) can be rewritten as

$$
\begin{aligned}
\dot{v}^{i}(t) & =A_{c} v^{i}(t) \\
v^{i}\left(t_{i, k}\right) & =y^{i}\left(t_{i, k}\right) \\
v_{j}^{i}\left(t_{j, k}\right) & =y_{j}^{j}\left(t_{j, k}\right), \quad j \in \mathcal{N}_{i} .
\end{aligned}
$$

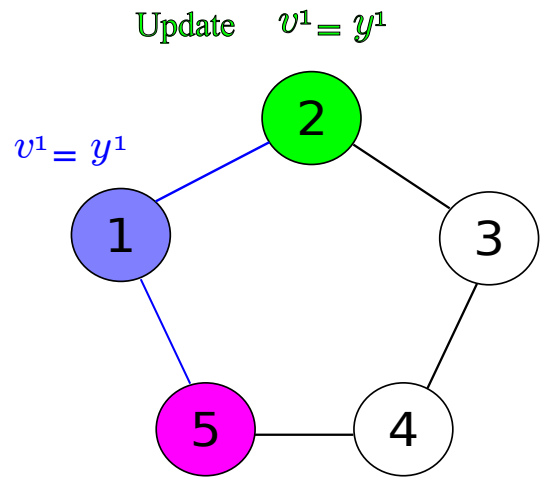

Update $v^{1}=y^{1}$

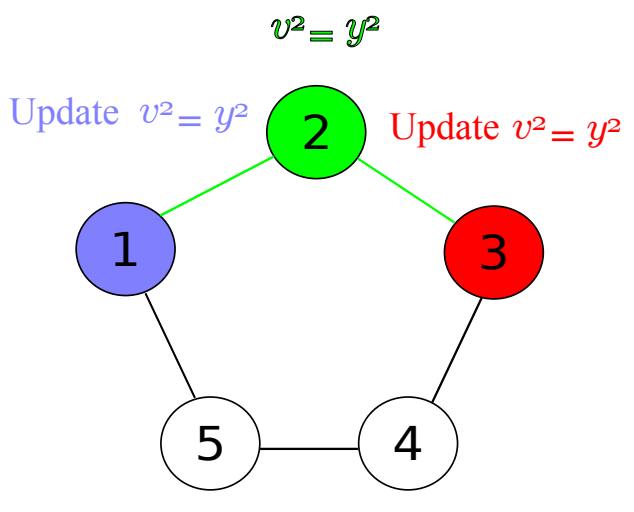

(b)

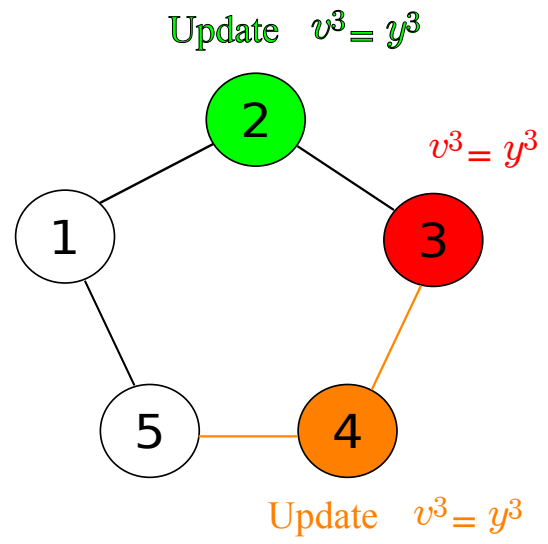

(c)

Figure 2: Update estimator $v^{i}$

Example 2. In Figure 2(a), the CTC is satisfied at $t_{1,1}$ for Agent 1. It updates its own estimate $y_{1}^{1}=x_{1}$, the first component of $T^{1}$ with $t_{1,1}$, and its own additional estimate $v^{1}=y^{1}$. Then, as shown in Figure 2(b), Agent 1 broadcasts $T^{1}$ and $y^{1}$. Its neighbours, Agents 2 and 5 , receive this message as seen in Figure 2(c). Agent 2, since the first component $t_{1,1}$ of $T^{1}$ is more recent than that of $T^{2}$, updates $y_{1}^{2}$ as $y_{1}^{2}=y_{1}^{1}$ and updates the additional estimate of Agent $1 v^{1}=y^{1}$. The first component of $T^{2}$ is now $t_{1,1}$. Agent 5 performs the same updates. Since there is no communication delay, the additional estimates $v^{1}$ evaluated by Agent 1,2 , and 5 are identical.

\section{Distributed event-triggered consensus}

Using the additional estimate $v^{i}$ introduced in Section 5.4, Theorem 2 in Section 6.1 introduces a CTC that can be evaluated by each agent in a distributed way. Section 6.2 introduces then an implementable distributed event-triggered consensus algorithm.

\subsection{CTC in distributed context}

As in Theorem 1, the initial states are considered to be known by all agents.In the experimental part, this condition will be relaxed: Agent $\mathrm{i}$ will initialize the state estimators for all other agents with its own value of the state. A communication is triggered at time $t=0$ to update the estimates of the neighbours of Agent $i$. All other agents behave in the same way.

Theorem 2. Assume that $(A, B)$ is controllable and that the communication graph is connected and undirected with a fixed topology described by the Laplacian matrix $L$. Consider some design parameter $\eta>0$. Agents which dynamics is (1) achieve a bounded consensus with

$$
\forall(i, j) \quad \lim _{t \rightarrow \infty}\left\|x_{i}-x_{j}\right\|^{2} \leq \frac{N^{3} \eta}{\beta \lambda_{\min }(P)}
$$


if the following condition on the perturbation bound is satisfied:

$$
S_{\max } \leq \sqrt{\frac{\alpha\left\|c_{2} \lambda_{2}(L) M\right\|}{\lambda_{\max }(P)}} \sqrt{\frac{N \eta}{\lambda_{\min }(P) \beta}}
$$

and if communication events are triggered when

$$
\tilde{\delta}_{i} \geq \rho z_{i}^{T} \Theta z_{i}+\eta
$$

with $\Theta_{i}=\left(2 c_{2}-b_{i} N_{i}\left(c_{2}-c\right)\right) M, 1 \geq \rho>0$, and

$$
\begin{aligned}
\tilde{\delta}_{i}= & c_{1}\left[\frac{1}{2 b_{i 2}}\left(z_{i}-N_{i} e_{i}^{i}\right)^{T} M\left(z_{i}-N_{i} e_{i}^{i}\right)\right. \\
& +\frac{b_{i 2}}{2} \sum_{j \in \mathcal{N}_{i}} N_{j}\left(y_{j}^{i}-v_{j}^{i}\right)^{T} M\left(y_{j}^{i}-v_{j}^{i}\right) \\
& +\left(z_{i}-N_{i} e_{i}^{i}\right)^{T} M \sum_{j \in \mathcal{N}_{i}}\left(v_{i}^{j}-y_{i}^{i}\right)+\frac{N_{i}}{2 b_{i}} e_{i}^{i T} M e_{i}^{i} \\
& +2\left(1+\frac{b_{i}}{2}\right) N_{i} \sum_{j \in \mathcal{N}_{i}}\left[\left(v_{i}^{j}-y_{i}^{i}\right)^{T} M\left(v_{i}^{j}-y_{i}^{i}\right)\right. \\
& \left.\left.+\left(y_{j}^{i}-v_{j}^{i}\right)^{T} M\left(y_{j}^{i}-v_{j}^{i}\right)\right]\right]+2\left(c_{2}-c\right) N_{i} z_{i}^{T} M e_{i}^{i} \\
& +\left[2 c\left(N_{i}\right)^{2}\left(1+b_{i}\right)+\frac{c_{2}-c}{b_{i}} N_{i}\right. \\
& \left.+c N_{i}(N-1)\left(b_{i}+\frac{3}{b_{i}}\right)\right] e_{i}^{i T} M e_{i}^{i}
\end{aligned}
$$

where $z_{i}=\sum_{j \in \mathcal{N}_{i}}\left(y_{i}^{i}-y_{j}^{i}\right), M=P B B^{T} P, 0<b_{i}<\frac{2 c_{2}}{\left(c-c_{2}\right) N_{i}}$ if $c_{2}>c, b_{i}>0$ otherwise.

The proof of Theorem 2 is in Appendix A.2 and the proof of absence of Zeno behavior in Appendix A.3.

The difference between Theorems 1 and 2 lies in the evaluation of the CTC. The term $\bar{\delta}_{i}$ in (10) has been replaced by $\tilde{\delta}_{i}$ in (28), which mainly depends on the discrepancy between the state estimates $y_{j}^{i}$ and the estimates of these state estimates $v_{j}^{i}$.

When an Agent $i$ broadcasts a message, the estimation error $e_{i}^{i}$ and the discrepancies $y_{j}^{i}-v_{j}^{i}$ and $v_{i}^{j}-y_{i}^{i}$ are reset according to (16), (22), and (21). As a consequence, the CTC (28) in Theorem 2 is no more satisfied.

\subsection{Summary of the distributed event-triggered consensus algorithm}

Results of Section 5 to 6 describing the proposed distributed event-triggered consensus approach are summarized in Algorithm 1 for some Agent $i$. This description is generic in the sense that all agents are controlled and trigger communications in the same way. The main loop of this algorithm is repeated until it is stopped by some external event.

\section{Example}

Consider a network of $N=5$ agents with unstable dynamics taken from [8] described by the following state and control matrices 


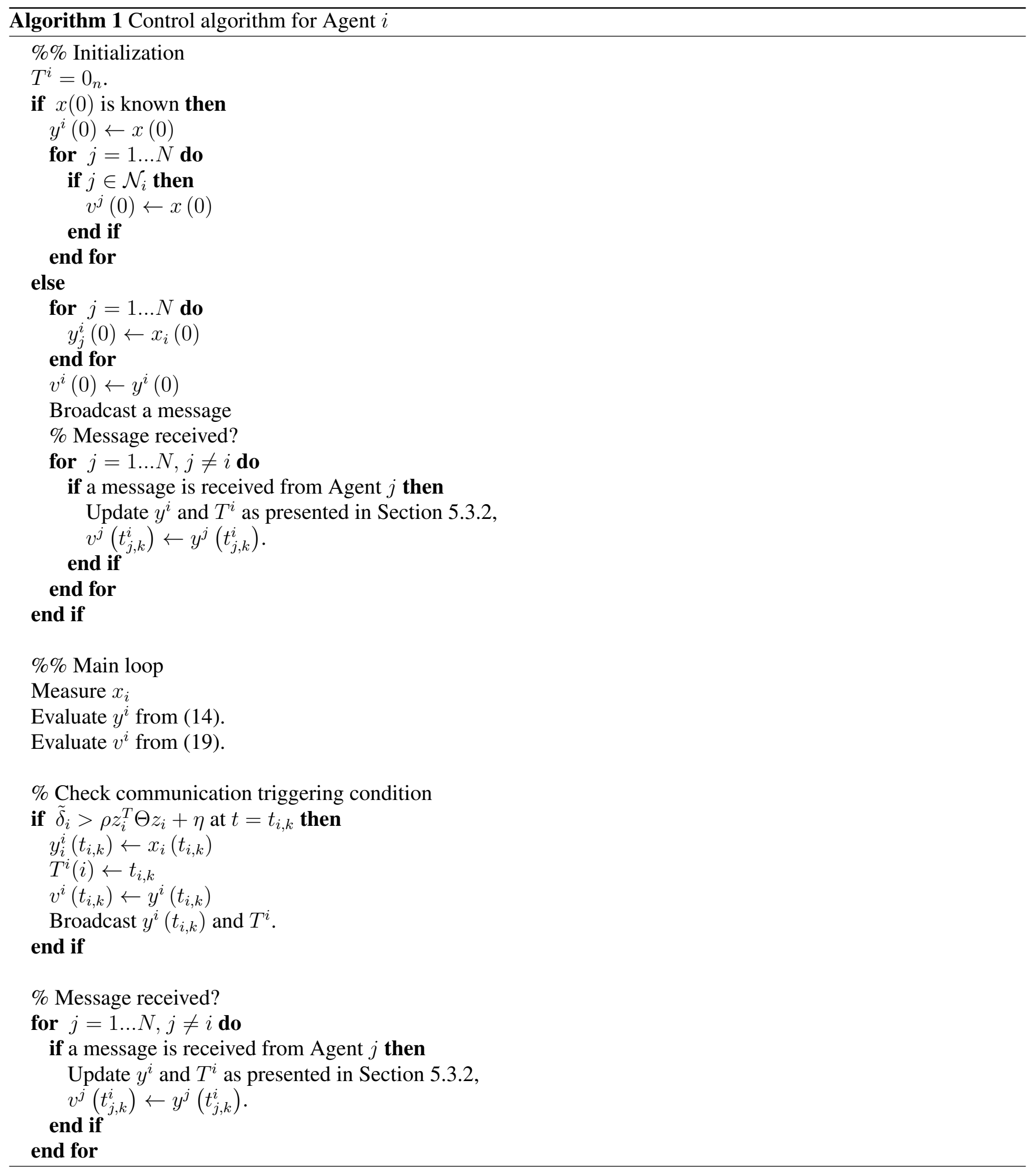




$$
A=\left[\begin{array}{ccc}
0.48 & 0.29 & -0.3 \\
0.13 & 0.23 & 0 \\
0 & -1.2 & -1
\end{array}\right] \quad B=\left[\begin{array}{cc}
2 & 0 \\
-1.5 & 1 \\
0 & 1
\end{array}\right]
$$

Solving (3) with $\alpha=1$, one obtains

$$
P=\left[\begin{array}{ccc}
4.8436 & 5.4783 & -1.1082 \\
5.4783 & 7.0514 & -1.4299 \\
-1.1082 & -1.4299 & 0.3778
\end{array}\right]
$$

The network topology is linear with Laplacian matrix

$$
L=\left[\begin{array}{ccccc}
1 & -1 & 0 & 0 & 0 \\
-1 & 2 & -1 & 0 & 0 \\
0 & -1 & 2 & -1 & 0 \\
0 & 0 & -1 & 2 & -1 \\
0 & 0 & 0 & -1 & 1
\end{array}\right]
$$

Each agent is assumed to know only its own state. The vector of initial states is

$$
\begin{aligned}
x(0)= & {\left[\left[\begin{array}{c}
8.5067 \\
-0.6568 \\
0
\end{array}\right]^{T}\left[\begin{array}{c}
1.7367 \\
-0.1855 \\
0
\end{array}\right]^{T}\left[\begin{array}{c}
-0.0340 \\
-0.4651 \\
0
\end{array}\right]^{T} \ldots\right.} \\
& \left.\cdots\left[\begin{array}{c}
-0.7768 \\
-0.3803 \\
0
\end{array}\right]^{T}\left[\begin{array}{c}
-0.6568 \\
1.5076 \\
0
\end{array}\right]^{T}\right]^{T} .
\end{aligned}
$$

The simulation duration is $\mathrm{T}=5 \mathrm{~s}$. Euler integration with a step $d t=0.01 \mathrm{~s}$ is used. As the system has been discretised, the minimum delay between the transmission of two messages by the same agent is set to $d t$. The perturbation $d(t)$ is assumed of constant value over each interval of the form $[k d t,(k+1) d t[$. The agent-specific component of $d(t)$ is $s_{i}(t)=\left[0, s_{i, 2}(t), 0\right]^{T}$ where $s_{i, 2}(t)$ is a zero-mean Gaussian noise with standard deviation $\sigma_{s}$, truncated at $S_{\max }=\sigma_{s}$ such that $\left|s_{i, 2}\right|=\left\|s_{i}\right\| \leq S_{\max }$. The common component of the perturbation is $m(t)=$ $\left[0, m_{2}(t), 0\right]^{T}$. Two cases are considered: a constant value $m_{2}(t)=M_{\max }$ (see, e.g., Figure 5 (a)) or a zero-mean Gaussian noise truncated at the standard deviation $\sigma_{m}$, such that $\left|m_{2}\right|=\|m\|<M_{\max }$ (Figure 5 (b)).

The parameters of the CTC are set as follows $\eta=0.1, c=\frac{1}{\lambda_{2}(L)}, c_{2}=0.1, b_{i}=1.36, b_{2 i}=1$ and $\rho=0.5$. The value of $c$ is imposed, that of $c_{2}$ is taken from [8]. The other values are chosen to reduce the number of required communications.

The proposed approach is compared to that of [8], evaluating in both cases the total number of messages broadcast $N_{\mathrm{m}} \leqslant \bar{N}_{\mathrm{m}}=N \mathrm{~T} / d t$. The residual communication ratio

$$
R_{\mathrm{com}}=100 \frac{N_{\mathrm{m}}}{\bar{N}_{\mathrm{m}}}
$$

of the number of broadcast messages is expressed in $\% . R_{\text {com }}$ indicates the proportions of time slots during which a communication has been triggered. It should be as small as possible. 

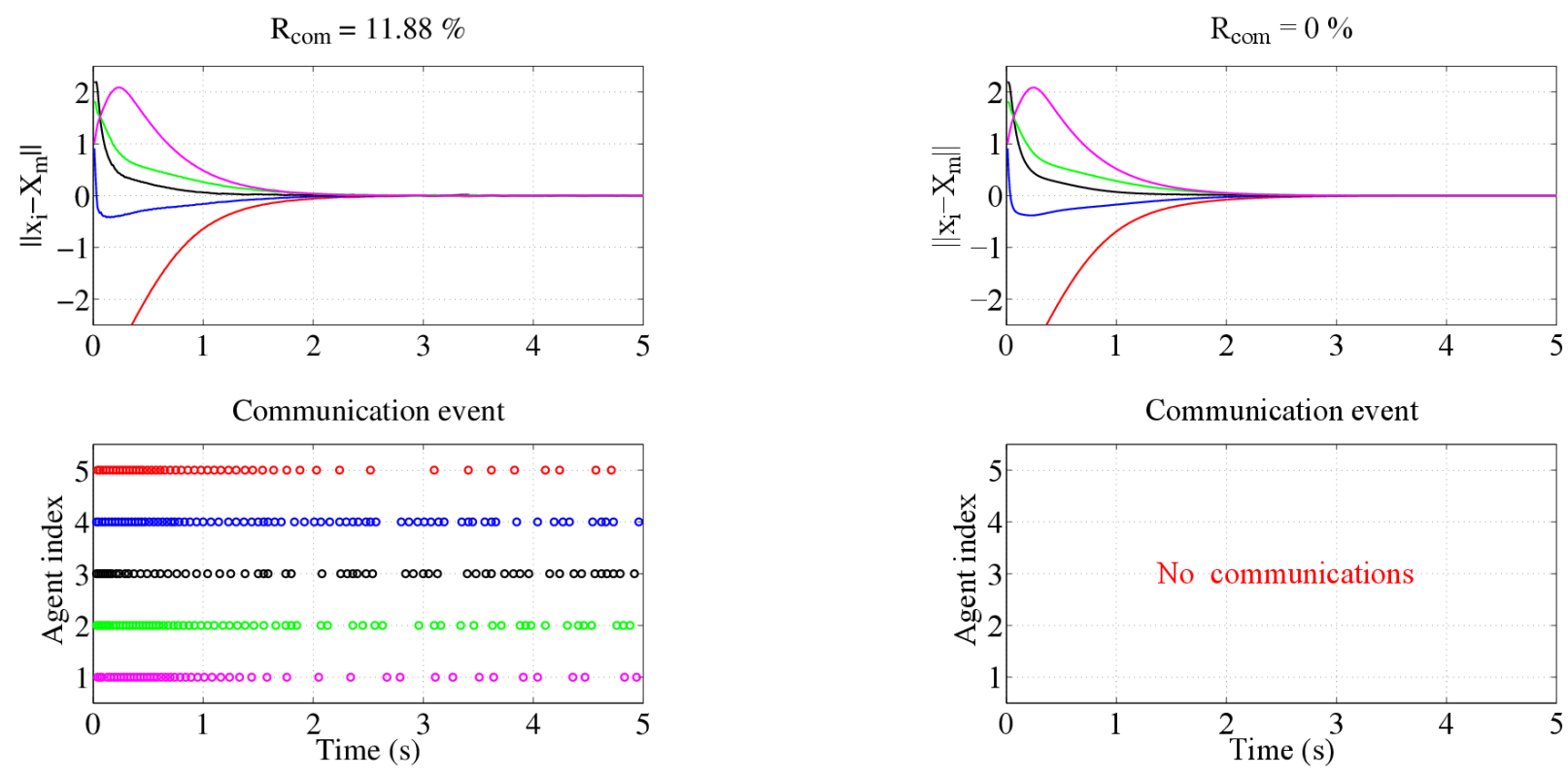

Error with respect to consensus and time instants of the broadcast messages when considering the reference estimator (13)

Error with respect to consensus and time instants of the broadcast messages when considering the proposed estimator(14).

Figure 3: Comparison between estimator (13) and new estimator (14) without perturbation. $X_{m}=\frac{1}{N} \sum_{i=1}^{N} x_{i}$. Initial state is known (Agent 1: magenta, Agent 2: green, Agent 3: black, Agent 4: blue, Agent 5: red).

\subsection{Without perturbation}

Figure 3 compares the performance in terms of consensus error and number of satisfied CTC for each agent, considering both estimators (13) and (14). When the initial conditions are perfectly known by all the agents and there is no perturbation, no communications are required when using the proposed estimator (14).

Figure 4 shows the results when each agent only knows its own initial state. When using the estimator (13) from [8], a first communication is enough to initialize the estimates of all agents, since each agent only estimates the states of its neighbours. With the proposed estimator (14), the CTCs are satisfied mainly at the beginning of the simulation. The delayed flooding method allows then an update of the estimates of all agents. After a short transient regime, only few communications are required.

\subsection{With perturbations}

Figure 5 shows the evolutions of $R_{\text {com }}$ as a function of $S_{\max }$ for different values of $M_{\max }$, when $m(t)$ is constant (Figure 5(a)) and when it is described by a truncated Gaussian (Figure 5(b)). With the considered parameters, the value of the upper-bound on $S_{\max }$ introduced in Theorem 2 is $S_{\max }=16.17$. With this value, the sufficient condition is satisfied for the following simulations, and one observes that a consensus is always reached.

When the level of perturbation is low the number of CTC satisfied for each agent is less with the proposed estimator (14) than with estimator (13). When $S_{\max }$ or $M_{\max }$ are large, the estimator (14) provides equivalent or even worse performance in terms of CTC compared to (13). This is mainly due to the additional terms introduced in the CTC (28). 

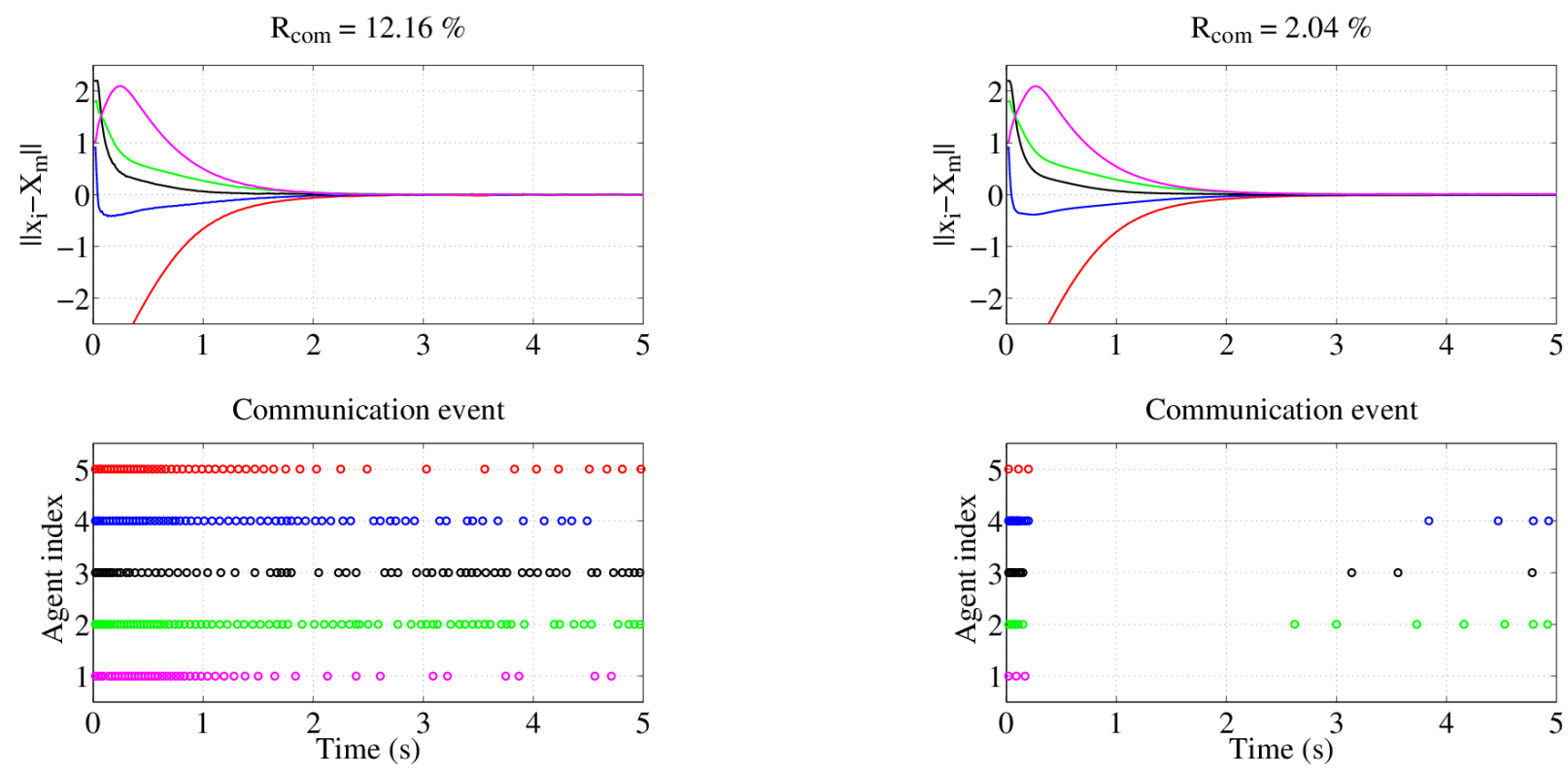

Error with respect to consensus and time instants of the broadcast messages when considering the reference estimator (13)

Error with respect to consensus and time instants of the broadcast messages when considering the proposed estimator (14)

Figure 4: Comparison between estimator (13) and new estimator (14) without perturbation. $X_{m}=\frac{1}{N} \sum_{i=1}^{N} x_{i}$. Initial state unknown. (Agent 1: magenta, Agent 2: green, Agent 3: black, Agent 4: blue, Agent 5: red).
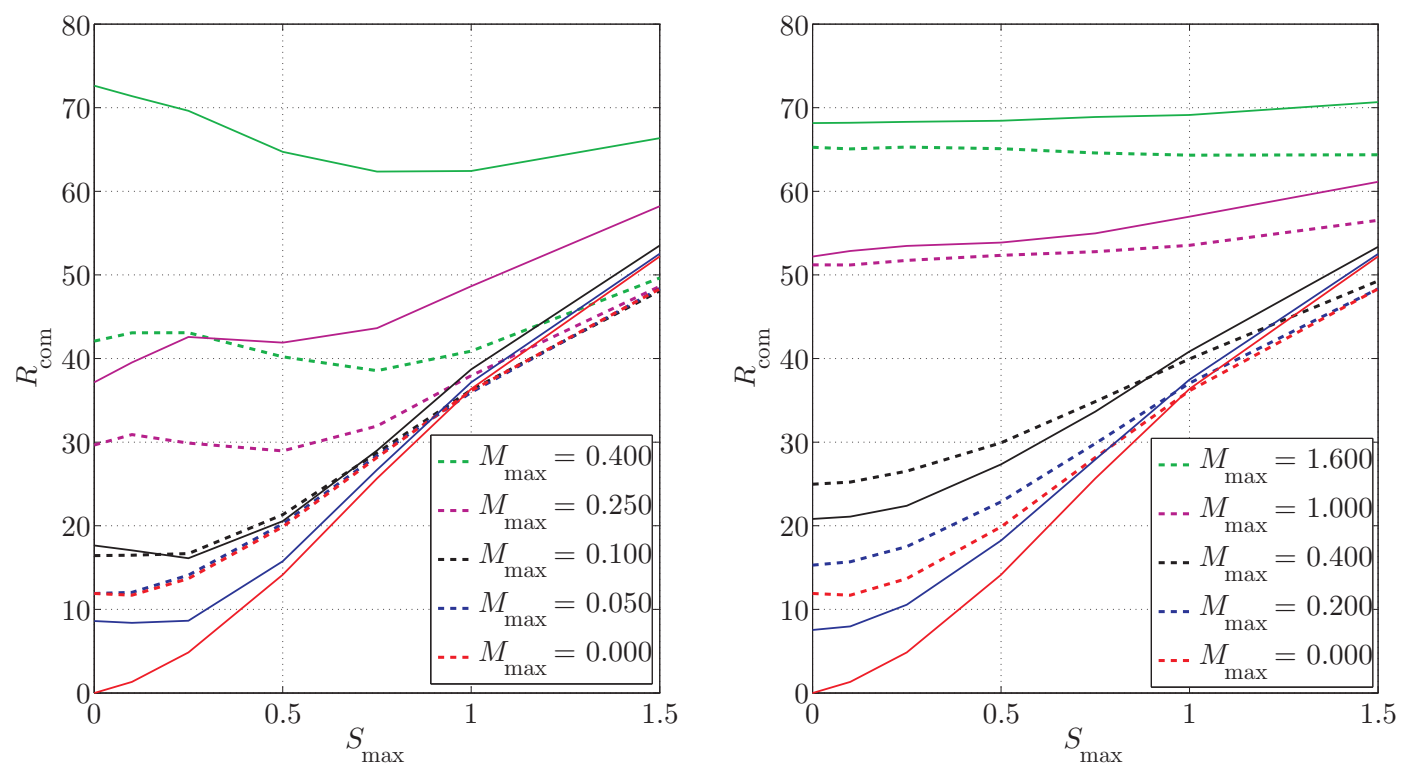

Figure 5: Evolution of $R_{\text {com }}$ as a function of $S_{\max }$ for different values of $M_{\max }$ when considering the reference estimator (13) (dashed) and the proposed estimator (14) (plain).

\subsection{Choosing $\eta$ in the CTC}

The parameter $\eta$ in the CTC allows to reach a compromise between the disagreement with respect to consensus and the reduction in the communication requirements. Figure 6 illustrates this compromise for

$$
\eta \in\{0.1, \quad 0.5, \quad 0.75,1,13.25,1.5,1.75,2,2.5\}
$$


and different values of $S_{\max }$ for the proposed estimator and that proposed by [8].

It can be seen that the proposed estimator outperforms that proposed by Garcia in terms of number of communications, while the order of magnitude of the consensus disagreement remains relatively close. Using the proposed estimator $R_{\text {com }}$ can be significantly reduced which is not the case using the reference estimator of Garcia up to

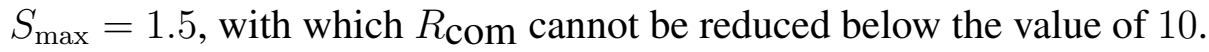

Figure 6 provides some guidelines to select the value of $\eta$ when communications constraints or when some bound on the disagreement with respect to consensus have to be satisfied.

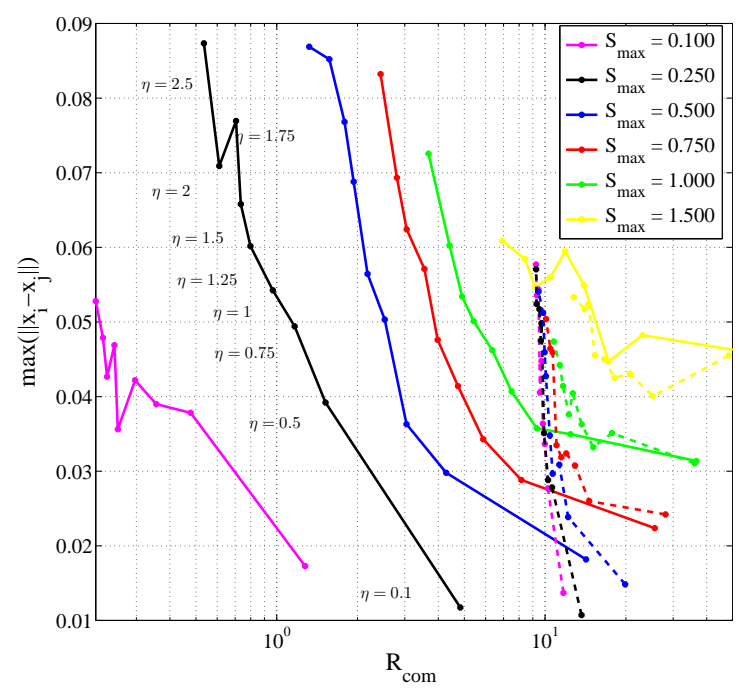

Figure 6: Evolution of $R_{\text {com }}$ and $\max _{i, j \in \mathcal{N}}\left(\left\|x_{i}-x_{j}\right\|\right)$ for different values of $\eta=$ $\{0.1,0.5,0.75,1,1.25,1.5,1.75,2,2.5\}$ with the reference estimator (13) (dashed) and the proposed estimator (14) (plain) for different values of $S_{\max }$. Each point corresponds to the mean value of 50 simulations.

\section{Conclusion}

This paper presents a distributed event-triggered communication technique to reach consensus in multi-agent systems with a reduced need for communication compared to state-of-the-art techniques. To obtain this result, each agent has to manage simultaneously two estimators of the states of the other agents in the network. The first provides an agent state estimate of all agents, which does not necessarily coincide among all agents. The second estimator considers only the neighbours of each agent and is less accurate but its value is constrained to coincide when two agents are neighbours. Both estimators are used to trigger communications. Convergence to consensus has been studied. Simulations have shown the effectiveness of the proposed estimators in presence of state perturbations when their level is moderate.

Extensions of this work will focus on the case of a time-varying topology and influence of packet drops during transmission of messages, and time delays in communications.

\section{Acknowledgments}

We thanks Direction Generale de l'Armement (DGA) and ICODE for a financial support to this study. 


\section{References}

[1] R. Aragues, J. Cortes, and C. Sagues. Distributed consensus algorithms for merging feature-based maps with limited communication. Robotics and Autonomous Systems, 59(3):163-180, 2011.

[2] Y. Cao and W. Ren. Distributed coordinated tracking with reduced interaction via a variable structure approach. IEEE Transactions on Automatic Control, 57(1):33-48, 2012.

[3] L. Cheng, Z. Hou, and M. Tan. A mean square consensus protocol for linear multi-agent systems with communication noises and fixed topologies. IEEE Transactions on Automatic Control, 59(1):261-267, 2014.

[4] J. Cortes and S. Martinez. Distributed Control of Robotic Networks. Princeton University Press, 2009.

[5] D. V. Dimarogonas, E. Frazzoli, and K. H. Johansson. Distributed event-triggered control for multi-agent systems. IEEE Transactions on Automatic Control, 57(5):1291 -1297, 2012.

[6] E. Garcia, Y. Cao, X. Wang, and D. W. Casbeer. Cooperative control with general linear dynamics and limited communication: Centralized and decentralized event-triggered control strategies. In American Control Conference (ACC), volume 1, pages 159-164, 2014.

[7] E Garcia, Y Cao, X Wang, and D. W. Casbeer. Cooperative control with general linear dynamics and limited communication: Periodic updates. In American Control Conference (ACC), volume 1, pages 3195-3200, 2014.

[8] E. Garcia, Y. Cao, X. Wang, and D. W. Casbeer. Decentralized event-triggered consensus with general linear dynamics. Automatica, 50(10):2633-2640, 2014.

[9] E. Garcia, Y. Cao, X. Wang, and D. W. Casbeer. Decentralized event-triggered consensus of linear multi-agent systems under directed graphs. American Control Conference (ACC), 1:5764-5769, 2015.

[10] J. He, P. Cheng, L. Shi, J. Chen, and Y. Sun. Time synchronization in WSNs: a maximum-value-based consensus approach. IEEE Transactions on Automatic Control, 59(3):660-675, 2014.

[11] W. R. Heinzelman, J. Kulik, and H. Balakrishnan. Adaptive protocols for information dissemination in wireless sensor networks. In In Proceedings of the 5th Annual ACM/IEEE International Conference on Mobile Computing and Networking, pages 174-185, 1999.

[12] A. Hu, J. Cao, M. Hu, and L. Guo. Event-triggered consensus of multi-agent systems with noises. Journal of The Franklin Institute, 352(9):3489-3503, 2014.

[13] S. Linsenmayer. Event-triggered control of multi-agent systems with double-integrator dynamics: Application to vehicle platooning and flocking algorithms. PhD thesis, KTH Royal Institute of Technology, Sweden, 2014.

[14] S. Linsenmayer and D. V. Dimarogonas. Event-triggered control for vehicle platooning. In American Control Conference (ACC), pages 3101-3106, 2015.

[15] R. Olfati-Saber, A. J. Fax, and R. M. Murray. Consensus and cooperation in networked multi-agent systems. Proceedings of the IEEE, 95(1):215-233, 2007.

[16] A Rahman, W Olesinski, and P Gburzynski. Controlled flooding in wireless ad-hoc networks. In Wireless Ad-Hoc Networks, 2004 International Workshop on, pages 73-78, 2004.

[17] L. Schenato and G. Gamba. A distributed consensus protocol for clock synchronization in wireless sensor network. In In 46th IEEE Conference on Decision and Control, pages 2289-2294. IEEE, 2007.

[18] G. S. Seyboth, D. V. Dimarogonas, and K. H. Johansson. Event-based broadcasting for multi-agent average consensus. Automatica, 49(1):245-252, 2013. 
[19] R. Wei. Consensus tracking under directed interaction topologies: Algorithms and experiments. In American Control Conference (ACC), volume 1, pages 742-747, 2008.

[20] W. Zhu, Z.P. Jiang, and G. Feng. Event-based consensus of multi-agent systems with general linear models. Automatica, 50(2):552-558, 2014.

\section{A. Appendix}

\section{A.1. Consensus convergence}

The system gathering the dynamics of all the agents is

$$
\dot{x}(t)=\bar{A} x(t)+\tilde{B} \tilde{y}(t)+d(t)
$$

where $x=\left[x_{1}^{T} \ldots x_{N}^{T}\right]^{T}, \bar{A}=1_{N} \otimes A, \tilde{B}=T\left(I_{N} \otimes \bar{B}_{1}\right), \bar{B}_{1}=c_{1} L \otimes B F, \tilde{y}=\left[y^{1 T} y^{2 T} \ldots y^{N T}\right]^{T} \in \mathbb{R}^{N^{2} n}$ is the vector gathering the estimates of the states of Agents $1, \ldots, N$ performed by all agents. Define $e=y-x \in \mathbb{R}^{N n}$. A matrix $T \in \mathbb{R}^{N n \times N^{2} n}$ is also defined to extract, from vector $\tilde{y}$, all terms $y_{k}^{k}, k=1 \ldots N$ :

$$
\begin{aligned}
T \tilde{y} & =T\left[y^{1 T} y^{2 T} \ldots y^{N T}\right]^{T} \\
& =\left[\begin{array}{ll}
y_{1}^{1 T} y_{2}^{2 T} \ldots y_{N}^{N T}
\end{array}\right]^{T} \\
& =y .
\end{aligned}
$$

This matrix can be expressed as $T=\left(\left(I_{N} \otimes 1_{N}^{T}\right) \circ\left(1_{N}^{T} \otimes I_{N}\right)\right) \otimes 1_{n}$, with $\circ$ the entrywise matrix product. One may easily show that $T \tilde{y}\left(1_{N} \otimes y\right)=y$.

Define the candidate Lyapunov function : $V=x^{T} \widehat{L} x$, with $\widehat{L}=L \otimes P$. Since the graph is undirected and $P$ is symmetric, $L$ and $\widehat{L}$ are symmetric and

$$
\dot{V}=2\left(x^{T} \widehat{L}(\bar{A} x+\tilde{B} \tilde{y})+d^{T}(t) \widehat{L} x\right)
$$

Define $\dot{V}_{1}=2 x^{T} \widehat{L}(\bar{A} x+\tilde{B} \tilde{y})$. The next section will show that $\dot{V}_{1}$ is upper bounded by $x^{T} \bar{L} x$. Then introduce $\dot{V}_{2}=x^{T} \bar{L} x+2 d^{T}(t) \widehat{L} x$, where one reminds that $\bar{L}=\widehat{L} A_{c}+A_{c}^{T} \widehat{L}$ and $A_{c}=\bar{A}+\bar{B}_{1}$. An upper bounds for $\dot{V}_{2}$, also evaluated in what follows, is then used to upper bound $\dot{V}$.

A.1.1. Upper bound for $\dot{V}_{1}$ : $\quad$ Let $\Delta_{i j}=y_{i}^{j}-y_{i}^{i}$ and define $\Delta(t)=\left[\Delta_{11}^{T}(t) \Delta_{12}^{T}(t) \ldots \Delta_{N, N-1}^{T}(t) \Delta_{N N}^{T}(t)\right]^{T} \in$ $\mathbb{R}^{N^{2} n}$. Note first that $\tilde{y}=1_{N} \otimes y+\Delta$ and $\tilde{e}=1_{N} \otimes e+\Delta$.

$$
\begin{aligned}
\dot{V}_{1} & =2 x^{T} \widehat{L}(\bar{A} x+\tilde{B} \tilde{y}) \\
& =2 x^{T} \widehat{L}\left(\bar{A} x+\tilde{B}\left(1_{N} \otimes y+\Delta\right)\right)
\end{aligned}
$$

Since $\tilde{B}=T\left(I_{N} \otimes \bar{B}_{1}\right), \bar{B}_{1}=c_{1} L \otimes(B F)$, and $T\left(1_{N} \otimes y\right)=y$, one obtains

$$
\begin{aligned}
\tilde{B}\left(1_{N} \otimes y\right) & =T\left(I_{N} \otimes \bar{B}_{1}\right)\left(1_{N} \otimes y\right) \\
& =T\left(I_{N} \otimes\left(\bar{B}_{1} y\right)\right) \\
& =\bar{B}_{1} y
\end{aligned}
$$


and

$$
\dot{V}_{1}=2 x^{T} \widehat{L}\left(\bar{A} x+\bar{B}_{1} y\right)+2 x^{T} \widehat{L} \tilde{B} \Delta
$$

Consider

$$
\dot{V}_{11}=2 x^{T} \widehat{L}\left(\bar{A} x+\bar{B}_{1} y\right)
$$

and

$$
\dot{V}_{12}=2 x^{T} \widehat{L} \tilde{B} \Delta \text {. }
$$

The expression of $\dot{V}_{11}$ can be found in [8], where it is shown that $\dot{V}_{11}=x^{T} \bar{L} x+\sum_{i=1}^{N}\left(\delta_{i}-z_{i}^{T} \Theta_{i} z_{i}\right)$ with

$$
\begin{aligned}
\delta_{i}= & 2\left(c_{2}-c\right) N_{i} z_{i}^{T} P B B^{T} P e_{i}^{i}+ \\
& {\left[2 c N_{i}^{2}\left(1+b_{i}\right)+\frac{c_{2}-c}{b_{i}} N_{i}\right.} \\
& \left.+c N_{i}(N-1)\left(b_{i}+\frac{3}{b_{i}}\right)\right] e_{i}^{i T} P B B^{T} P e_{i}^{i} .
\end{aligned}
$$

Using the expression of $\tilde{B}$ and $\bar{B}_{1}$, and the fact that $e=y-x, \dot{V}_{12}$ may be rewritten as

$$
\dot{V}_{12}=2(\widehat{L}(y-e))^{T} T\left(I_{N} \otimes\left(c_{1} L \otimes(B F)\right)\right) \Delta .
$$

Using the property of $T$,

$$
T\left(I_{N} \otimes\left(c_{1} L \otimes(B F)\right)\right) \Delta=\left[\begin{array}{c}
c_{1} B F \sum_{k \in \mathcal{N}_{1}}\left(\Delta_{11}-\Delta_{1 k}\right) \\
\vdots \\
c_{1} B F \sum_{k \in \mathcal{N}_{N}}\left(\Delta_{N N}-\Delta_{N k}\right)
\end{array}\right] .
$$

Since $\Delta_{i i}=0, \widehat{L}=L \otimes P$, and $F=-B^{T} P$ one may rewrite $\dot{V}_{12}$ as

$$
\begin{aligned}
\dot{V}_{12}= & c_{1} \sum_{i=1}^{N}\left[\sum_{j \in \mathcal{N}_{i}}\left(y_{i}^{i}-y_{j}^{j}\right)^{T}\left(-P B B^{T} P\right) \sum_{k \in \mathcal{N}_{i}}\left(-\Delta_{i k}\right)\right. \\
& \left.-\sum_{j \in \mathcal{N}_{i}}\left(e_{i}^{i}-e_{j}^{j}\right)^{T}\left(-P B B^{T} P\right) \sum_{k \in \mathcal{N}_{i}}\left(-\Delta_{i k}\right)\right]
\end{aligned}
$$

One may rewrite $\sum_{j \in \mathcal{N}_{i}}\left(y_{i}^{i}-y_{j}^{j}\right)^{T}$ as

$$
\begin{aligned}
\sum_{j \in \mathcal{N}_{i}}\left(y_{i}^{i}-y_{j}^{j}\right)^{T} & =\sum_{j \in \mathcal{N}_{i}}\left(y_{i}^{i}-y_{j}^{i}+\left(y_{j}^{i}-y_{j}^{j}\right)\right)^{T} \\
& =z_{i}^{T}+\sum_{j \in \mathcal{N}_{i}} \Delta_{j i}^{T}
\end{aligned}
$$

Inserting this expression in (37) and defining $M=P B B^{T} P$, one gets

$$
\begin{aligned}
\dot{V}_{12}= & c_{1} \sum_{i=1}^{N}\left[z_{i}^{T} M \sum_{k \in \mathcal{N}_{i}} \Delta_{i k}+\sum_{j \in \mathcal{N}_{i}}\left(\Delta_{j i}\right)^{T} M \sum_{k \in \mathcal{N}_{i}} \Delta_{i k}\right. \\
& \left.-N_{i} e_{i}^{i T} M \sum_{k \in \mathcal{N}_{i}} \Delta_{i k}+\sum_{j \in \mathcal{N}_{i}} e_{j}^{j T} M \sum_{k \in \mathcal{N}_{i}} \Delta_{i k}\right] .
\end{aligned}
$$


Using $x^{T} y \leq \frac{1}{2 b_{i}} x^{T} x+\frac{b_{i}}{2} y^{T} y$ for any $b_{i}>0$, one obtains

$$
\begin{aligned}
\sum_{j \in \mathcal{N}_{i}}\left(\Delta_{j i}\right)^{T} M \sum_{k \in \mathcal{N}_{i}} \Delta_{i k} & =\sum_{j \in \mathcal{N}_{i}} \sum_{k \in \mathcal{N}_{i}}\left(\Delta_{j i}\right)^{T} M \Delta_{i k} \\
& \leq \sum_{j \in \mathcal{N}_{i}} \sum_{k \in \mathcal{N}_{i}}\left[\frac{1}{2 b_{i 1}} \Delta_{i j}^{T} M \Delta_{i j}+\frac{b_{i 1}}{2} \Delta_{i k}^{T} M \Delta_{i k}\right] \\
& \leq \sum_{j \in \mathcal{N}_{i}} N_{i}\left[\left(\frac{1}{2 b_{i 1}}+\frac{b_{i 1}}{2}\right) \Delta_{i j}^{T} M \Delta_{i j}\right]
\end{aligned}
$$

and

$$
\begin{aligned}
\sum_{j \in \mathcal{N}_{i}} e_{j}^{j T} M \sum_{k \in \mathcal{N}_{i}} \Delta_{i k} & =\sum_{j \in \mathcal{N}_{i}} \sum_{k \in \mathcal{N}_{i}} e_{j}^{j T} M \Delta_{i k} \\
& \leq \sum_{j \in \mathcal{N}_{i}} \sum_{k \in \mathcal{N}_{i}}\left[\frac{1}{2 b_{i}} e_{j}^{j T} M e_{j}^{j}+\frac{b_{i}}{2} \Delta_{i k}^{T} M \Delta_{i k}\right] \\
& \leq \sum_{j \in \mathcal{N}_{i}} N_{i}\left[\frac{1}{2 b_{i}} e_{j}^{j T} M e_{j}^{j}+\frac{b_{i}}{2} \Delta_{i j}^{T} M \Delta_{i j}\right]
\end{aligned}
$$

Using these upper bounds in (38), one gets

$$
\begin{aligned}
\dot{V}_{12} \leq & c_{1} \sum_{i=1}^{N}\left[\left(z_{i}-N_{i} e_{i}^{i}\right)^{T} M \sum_{k \in \mathcal{N}_{i}} \Delta_{i k}+N_{i} \sum_{j \in \mathcal{N}_{i}}\left(\frac{1}{2 b_{i 1}}+\frac{b_{i 1}}{2}\right)\right. \\
& \left.\times \Delta_{i j}^{T} M \Delta_{i j}+\sum_{j \in \mathcal{N}_{i}} N_{i}\left[\frac{1}{2 b_{i}} e_{j}^{j T} M e_{j}^{j}+\frac{b_{i}}{2} \Delta_{i j}^{T} M \Delta_{i j}\right]\right] .
\end{aligned}
$$

Choosing $b_{i 1}=1$, one gets

$$
\begin{aligned}
\dot{V}_{12} \leq & c_{1} \sum_{i=1}^{N}\left[\left(z_{i}-N_{i} e_{i}^{i}\right)^{T} M \sum_{k \in \mathcal{N}_{i}} \Delta_{i k}+\frac{N_{i}}{2 b_{i}} e_{i}^{i T} M e_{i}^{i}\right. \\
& \left.+\sum_{j \in \mathcal{N}_{i}} N_{i}\left(1+\frac{b_{i}}{2}\right) \Delta_{i j}^{T} M \Delta_{i j}\right]
\end{aligned}
$$

Inserting (39) in $\dot{V}_{1}$ and one obtains

$$
\dot{V}_{1} \leq x^{T} \bar{L} x+\sum_{i=1}^{N}\left(\bar{\delta}_{i}-\sigma z_{i}^{T} \Theta_{i} z_{i}\right)
$$

where

$$
\begin{aligned}
\bar{\delta}_{i}= & c_{1}\left[\left(z_{i}-N_{i} e_{i}^{i}\right)^{T} M \sum_{j \in \mathcal{N}_{i}}\left(y_{i}^{j}-y_{i}^{i}\right)+\frac{N_{i}}{2 b_{i}} e_{i}^{i T} M e_{i}^{i}\right. \\
+ & \left.\left(1+\frac{b_{i}}{2}\right) N_{i} \sum_{j \in \mathcal{N}_{i}}\left(\left(y_{i}^{j}-y_{i}^{i}\right)^{T} M\left(y_{i}^{j}-y_{i}^{i}\right)\right)\right]+\delta_{i} .
\end{aligned}
$$

Since $\bar{L}$ is semi-definite negative, $\dot{V}_{1} \leq 0$ if, for $i, j=1 \ldots N$, the communication events are triggered when $\bar{\delta}_{i}>\rho z_{i}^{T} \Theta z_{i}$ with $0<\rho \leq 1$. 
Remark 3. With $\delta_{i}>\rho z_{i}^{T} \Theta z_{i}$ and no perturbation, $V_{1}(t)$ converges asymptotically to zero. In order to reduce the number of broadcast communications, a threshold $\eta$ can be introduced so that $\bar{\delta}_{i} \geq \rho z_{i}^{T} \Theta z_{i}+\eta$.

A.1.2. Upper bound for $V$ : Assuming that there is no perturbation, one is now interested in bounding $\left\|x_{i}-x_{j}\right\|$ when the CTC (28) is satisfied.

First note that $x^{T} \widehat{L} x \geq 0$, so

$$
x^{T} \widehat{L} x \leq \lambda_{\max }(\widehat{L}) x^{T} x
$$

and that $x^{T} \bar{L} x \leq 0$, so

$$
-x^{T} \bar{L} x \geq \lambda_{\min >0}(-\bar{L}) x^{T} x .
$$

Combining these results, one obtains

$$
x^{T} \widehat{L} x \frac{1}{\lambda_{\max }(\widehat{L})} \leq x^{T} x \leq-x^{T} \bar{L} x \frac{1}{\lambda_{\min >0}(-\bar{L})}
$$

and thus

$$
x^{T} \bar{L} x \leq-\beta x^{T} \widehat{L} x
$$

where $\beta=\frac{\lambda_{\min >0}(-\bar{L})}{\lambda_{\max }(\widehat{L})}$.

With the triggering condition defined in Theorem 1, one obtains

$$
\begin{aligned}
\dot{V}(t) & \leq x^{T} \bar{L} x+\sum_{i=1}^{N}\left(\delta_{i}-\rho z_{i}^{T} \Theta_{i} z_{i}\right) \\
& \leq-\beta V(t)+N \eta
\end{aligned}
$$

from which one deduces that $V(t) \leq V(0) e^{-\beta t}+\frac{N \eta}{\beta}$. Consequently,

$$
\lim _{t \rightarrow \infty} V(t) \leq \frac{N \eta}{\beta}
$$

$V(t)$ may be rewritten as

$$
\begin{aligned}
V(t) & =x^{T} \widehat{L} x \\
& =\sum_{i=1}^{N}\left(x_{i}^{T} P \sum_{k \in \mathcal{N}_{i}}\left(x_{i}-x_{k}\right)\right) \\
& =\sum_{i=1}^{N} \sum_{k \in \mathcal{N}_{i}}\left(x_{i}^{T} P\left(x_{i}-x_{k}\right)\right) \\
& =\frac{1}{2} \sum_{i=1}^{N} \sum_{k \in \mathcal{N}_{i}} 2\left(x_{i}^{T} P x_{i}-x_{i}^{T} P x_{k}\right)
\end{aligned}
$$

As the graph is undirected, $V(t)$ becomes

$$
\begin{aligned}
V(t) & =\frac{1}{2} \sum_{i=1}^{N} \sum_{k \in \mathcal{N}_{i}}\left(x_{i}^{T} P x_{i}-2 x_{i}^{T} P x_{k}+x_{k}^{T} P x_{k}\right) \\
& =\frac{1}{2} \sum_{i=1}^{N} \sum_{k \in \mathcal{N}_{i}}\left(x_{i}-x_{k}\right)^{T} P\left(x_{i}-x_{k}\right) .
\end{aligned}
$$


Since the graph is connected, each term $\left(x_{i}-x_{k}\right)^{T} P\left(x_{i}-x_{k}\right)$ appears twice in (43). Thus, from (42) and (43), one has

$$
\begin{aligned}
\lim _{t \rightarrow \infty} V(t) & \leq \frac{N \eta}{\beta} \\
\lim _{t \rightarrow \infty} \sum_{i=1}^{N} \sum_{k \in \mathcal{N}_{i}}\left\|x_{i}-x_{k}\right\|^{2} \lambda_{\min }(P) & \leq \frac{N \eta}{\beta} \\
\forall k \in \mathcal{N}_{i}, \quad \lim _{t \rightarrow \infty}\left\|x_{i}-x_{k}\right\|^{2} & \leq \frac{N \eta}{\beta \lambda_{\min }(P)}
\end{aligned}
$$

The graph is connected, thus for any pair of agents $(i, j)$, there exists a path between them linking neighboring agents, the indexes of these agents are $k_{1}, k_{2}, \ldots, k_{m}$ and

$$
\begin{aligned}
\left\|x_{i}-x_{j}\right\| & \leq\left\|x_{i}-x_{k_{1}}\right\|+\left\|x_{k_{1}}-x_{k_{2}}\right\|+\ldots+\left\|x_{k_{m}}-x_{j}\right\| \\
& \leq N \max _{k \in \mathcal{N}, \ell \in \mathcal{N}_{k}}\left\|x_{k}-x_{\ell}\right\| .
\end{aligned}
$$

Combining (44) and (45), one gets

$$
\forall(i, j) \in \mathcal{N}, \quad \lim _{t \rightarrow \infty}\left\|x_{i}-x_{j}\right\|^{2} \leq \frac{N^{3} \eta}{\beta \lambda_{\min }(P)}
$$

The perturbations terms do not appear in $\bar{\delta}_{i}$ and $\Theta_{i}$, but they impact the estimation error and the communication triggering frequency.

A.1.3. Upper bound for $\dot{V}_{2}$ : Since $L 1_{N}=0$ one has

$$
(L \otimes P)\left(1_{N} \otimes m\right)=\left(\left(L 1_{N}\right) \otimes(P m)\right)=0
$$

and one deduces

$$
\begin{aligned}
\dot{V}_{2} & =2 x^{T} \widehat{L} d+x^{T} \bar{L} x \\
& =2 x^{T}(L \otimes P)\left(1_{N} \otimes m+s\right)+x^{T} \bar{L} x \\
& =2 x^{T} \widehat{L} s+x^{T} \bar{L} x .
\end{aligned}
$$

Let $\dot{V}_{21}=2 x^{T} \widehat{L} s$ and $\dot{V}_{22}=x^{T} \bar{L} x$. Then, considering a sequence of $b_{i}>0, i=1, \ldots, N$, one has

$$
\begin{aligned}
\dot{V}_{21} & =2 \sum_{i=1}^{N}\left(\sum_{j \in \mathcal{N}_{i}}\left(x_{i}-x_{j}\right)^{T} P s_{i}\right) \\
& \leq 2 \sum_{i=1}^{N} \sum_{j \in \mathcal{N}_{i}}\left(\frac{b_{i}}{2}\left(x_{i}-x_{j}\right)^{T} P\left(x_{i}-x_{j}\right)+\frac{1}{2 b_{i}} s_{i}^{T} P s_{i}\right) \\
& \leq \sum_{i=1}^{N} \sum_{j \in \mathcal{N}_{i}}\left(b_{i}\left(x_{i}-x_{j}\right)^{T} P\left(x_{i}-x_{j}\right)+\frac{1}{b_{i}} \lambda_{\max }(P) S_{\max }^{2}\right)
\end{aligned}
$$


To bound $\dot{V}_{22}$, using the expression of $\bar{L}$, one gets

$$
\dot{V}_{22}=x^{T}\left[L \otimes\left(P A+A^{T} P\right)-(L L) \otimes\left(2 c_{1} P B B^{T} P\right)\right] x
$$

Since $M=P B B^{T} P$ and $L$ are symmetric semi-define positive matrices, one obtains

$$
\begin{aligned}
x^{T}(L \otimes M) x & =x^{T}\left(I_{N} \otimes\left(B^{T} P\right)\right)^{T}\left(L \otimes I_{n}\right)\left(I_{N} \otimes\left(B^{T} P\right)\right) x \\
& =x^{T}\left(I_{N} \otimes\left(B^{T} P\right)\right)^{T} U^{T} \Lambda U\left(I_{N} \otimes\left(B^{T} P\right)\right) x
\end{aligned}
$$

where $\Lambda$ is a diagonal matrix with elements $\Lambda_{i}=\lambda_{i}\left(L \otimes I_{n}\right), i=1 \ldots N n$, and $U$ is the matrix of corresponding eigenvectors.

Introducing $q=U\left(I_{N} \otimes\left(B^{T} P\right)\right) x$, one obtains

$$
\begin{aligned}
x^{T}(L \otimes M) x & =q^{T} \Lambda q \\
& =\sum_{i=1}^{N n} q_{i}^{2} \lambda_{i}\left(L \otimes I_{n}\right) \\
& \leq \frac{1}{\lambda_{\min >0}\left(L \otimes I_{n}\right)} \sum_{i=1}^{N n} q_{i}^{2} \lambda_{i}\left(L \otimes I_{n}\right)^{2} .
\end{aligned}
$$

Since $\lambda_{\min >0}\left(L \otimes I_{n}\right)=\lambda_{\min >0}(L) \lambda_{\min >0}\left(I_{n}\right)=\lambda_{2}(L)$, one obtains

$$
\begin{aligned}
x^{T}(L \otimes M) x & \leq \frac{1}{\lambda_{2}(L)} q^{T} \Lambda^{2} q \\
& \leq \frac{1}{\lambda_{2}(L)} x^{T}\left(I_{N} \otimes\left(B^{T} P\right)\right)^{T}\left((L L) \otimes I_{n}\right)\left(I_{N} \otimes\left(B^{T} P\right)\right) x \\
& \leq \frac{1}{\lambda_{2}(L)} x^{T}((L L) \otimes M) x
\end{aligned}
$$

and thus

$$
-x^{T}((L L) \otimes M) x \leq-\lambda_{2}(L) x^{T}(L \otimes M) x .
$$

Injecting (47) in $\dot{V}_{22}$, one gets

$$
\begin{aligned}
\dot{V}_{22} & =x^{T} \bar{L} x \\
& =x^{T}\left[L \otimes\left(P A+A^{T} P\right)-2 c_{1}(L L) \otimes M\right] x \\
& \leq x^{T}\left[L \otimes\left(P A+A^{T} P-2 c_{1} M \lambda_{2}(L)\right)\right] x
\end{aligned}
$$

Reminding that $c_{1}=c+c_{2}$ and $c=\frac{1}{\lambda_{2}(L)}$, one gets

$$
\begin{aligned}
\dot{V}_{22} & \leq x^{T}\left[L \otimes\left(P A+A^{T} P-2\left(\frac{1}{\lambda_{2}(L)}+c_{2}\right) M \lambda_{2}(L)\right)\right] x \\
& \leq x^{T}\left[L \otimes\left(P A+A^{T} P-2 M-2 c_{2} M \lambda_{2}(L)\right)\right] x .
\end{aligned}
$$

Using (3), one obtains

$$
\dot{V}_{22} \leq x^{T}\left[L \otimes\left(-2 \alpha P-2 c_{2} \lambda_{2}(L) M\right)\right] x
$$


and using (43), $\dot{V}_{22}$ becomes

$$
\dot{V}_{22} \leq \frac{1}{2} \sum_{i=1}^{N} \sum_{j \in \mathcal{N}_{i}}\left[\left(x_{i}-x_{j}\right)^{T}\left(-2 \alpha P-2 c_{2} \lambda_{2}(L) M\right)\left(x_{i}-x_{j}\right)\right]
$$

Since $\dot{V}_{2}=\dot{V}_{21}+\dot{V}_{22}$, combining (46) and (48), one gets

$$
\begin{aligned}
\dot{V}_{2} \leq & \sum_{i=1}^{N}\left(\sum_{j \in \mathcal{N}_{i}}\left[\left(x_{i}-x_{j}\right)^{T}\left(\left(b_{i}-\alpha\right) P-c_{2} \lambda_{2}(L) M\right)\left(x_{i}-x_{j}\right)\right]\right. \\
& \left.+\frac{N_{i}}{b_{i}} \lambda_{\max }(P) S_{\max }^{2}\right) .
\end{aligned}
$$

One now searches a condition on $S_{\max }$ to ensure that $V_{2}$ is decreasing. Having $\dot{V}_{2} \leq 0$ is equivalent to

$$
\sum_{i=1}^{N}\left(\frac{N_{i}}{b_{i}} \lambda_{\max }(P) S_{\max }^{2}\right) \leq \sum_{i=1}^{N} \sum_{j \in \mathcal{N}_{i}}\left[\left(x_{i}-x_{j}\right)^{T}\left(\left(\alpha-b_{i}\right) P+c_{2} \lambda_{2}(L) M\right)\left(x_{i}-x_{j}\right)\right] .
$$

Sufficient conditions to satisfy (50) are for $i=1, \ldots, N$

$$
\frac{N_{i}}{b_{i}} \lambda_{\max }(P) S_{\max }^{2} \leq \sum_{j \in \mathcal{N}_{i}}\left[\left(x_{i}-x_{j}\right)^{T}\left(\left(\alpha-b_{i}\right) P+c_{2} \lambda_{2}(L) M\right)\left(x_{i}-x_{j}\right)\right] .
$$

Each inequality (51) is satisfied if the following condition holds

$$
\frac{N_{i}}{b_{i}} \lambda_{\max }(P) S_{\max }^{2} \leq \sum_{j \in \mathcal{N}_{i}}\left[\left(x_{i}-x_{j}\right)^{T}\left(x_{i}-x_{j}\right)\left\|\left(\alpha-b_{i}\right) P+c_{2} \lambda_{2}(L) M\right\|\right]
$$

with $\|M\|=\max _{i=1: n}\left(\left|\lambda_{i}(M)\right|\right)$. This provides an upper bound for $S_{\max }$

$$
S_{\max }^{2} \leq \sum_{j \in \mathcal{N}_{i}}\left\|x_{i}-x_{j}\right\|^{2} \frac{b_{i}\left\|\left(\alpha-b_{i}\right) P+c_{2} \lambda_{2}(L) M\right\|}{\lambda_{\max }(P) N_{i}} .
$$

Using (44) in (52), one gets

$$
\begin{aligned}
S_{\max }^{2} & \leq \frac{b_{i}\left\|\left(\alpha-b_{i}\right) P+c_{2} \lambda_{2}(L) M\right\|}{\lambda_{\max }(P) N_{i}} \frac{N_{i} N \eta}{\beta \lambda_{\min }(P)} \\
S_{\max } & \leq \sqrt{\frac{b_{i}\left\|\left(\alpha-b_{i}\right) P+c_{2} \lambda_{2}(L) M\right\|}{\lambda_{\max }(P)}} \sqrt{\frac{N \eta}{\lambda_{\min }(P) \beta}} .
\end{aligned}
$$

Choosing $b_{i}=\alpha$, one obtains

$$
S_{\max } \leq \sqrt{\frac{\alpha\left\|c_{2} \lambda_{2}(L) M\right\|}{\lambda_{\max }(P)}} \sqrt{\frac{N \eta}{\lambda_{\min }(P) \beta}}
$$

Using (53) in (49), one finally gets $\dot{V}_{2} \leq 0$, which leads to $\dot{V} \leq 0$. The system converges thus to a bounded consensus. 


\section{A.2. Proof of Theorem 2}

Starting from (39) in Appendix A.1.1, one has

$$
\begin{aligned}
\dot{V}_{12} \leq & c_{1} \sum_{i=1}^{N}\left[\left(z_{i}-N_{i} e_{i}^{i}\right)^{T} M \sum_{j \in \mathcal{N}_{i}}\left(y_{i}^{j}-y_{i}^{i}\right)+\frac{N_{i}}{2 b_{i}} e_{i}^{i T} M e_{i}^{i}\right. \\
& \left.+\left(1+\frac{b_{i}}{2}\right) N_{i} \sum_{j \in \mathcal{N}_{i}}\left(\Delta_{i j}^{T} M \Delta_{i j}\right)\right] .
\end{aligned}
$$

Expressing $\Delta_{i j}$ as $\Delta_{i j}=y_{i}^{j}-v_{i}^{j}+v_{i}^{j}-y_{i}^{i}$ in (54), one gets

$$
\begin{aligned}
\dot{V}_{12} \leq & c_{1} \sum_{i=1}^{N}\left[\left(z_{i}-N_{i} e_{i}^{i}\right)^{T} M \sum_{j \in \mathcal{N}_{i}}\left(y_{i}^{j}-v_{i}^{j}\right)\right. \\
& +\left(z_{i}-N_{i} e_{i}^{i}\right)^{T} M \sum_{j \in \mathcal{N}_{i}}\left(v_{i}^{j}-y_{i}^{i}\right) \\
& \left.+\frac{N_{i}}{2 b_{i}} e_{i}^{i T} M e_{i}^{i}+\left(1+\frac{b_{i}}{2}\right) N_{i} \sum_{j \in \mathcal{N}_{i}}\left(\Delta_{i j}^{T} M \Delta_{i j}\right)\right]
\end{aligned}
$$

Using $x y \leq \frac{1}{2 b_{i 2}} x^{T} x+\frac{b_{i 2}}{2} y^{T} y$, with $b_{i 2}>0$,

$$
\begin{aligned}
\dot{V}_{12} \leq & c_{1} \sum_{i=1}^{N}\left[\frac{1}{2 b_{i 2}}\left(z_{i}-N_{i} e_{i}^{i}\right)^{T} M\left(z_{i}-N_{i} e_{i}^{i}\right)\right. \\
& +\frac{b_{i 2}}{2} \sum_{j \in \mathcal{N}_{i}}\left(y_{i}^{j}-v_{i}^{j}\right)^{T} M \sum_{j \in \mathcal{N}_{i}}\left(y_{i}^{j}-v_{i}^{j}\right) \\
& +\left(z_{i}-N_{i} e_{i}^{i}\right)^{T} M \sum_{j \in \mathcal{N}_{i}}\left(v_{i}^{j}-y_{i}^{i}\right)+\frac{N_{i}}{2 b_{i}} e_{i}^{i T} M e_{i}^{i} \\
& \left.+\left(1+\frac{b_{i}}{2}\right) N_{i} \sum_{j \in \mathcal{N}_{i}}\left(\Delta_{i j}^{T} M \Delta_{i j}\right)\right]
\end{aligned}
$$

Let

$$
\begin{aligned}
& \dot{V}_{12 a}=\sum_{i=1}^{N} \sum_{j \in \mathcal{N}_{i}}\left(y_{i}^{j}-v_{i}^{j}\right)^{T} M \sum_{j \in \mathcal{N}_{i}}\left(y_{i}^{j}-v_{i}^{j}\right) \\
& \dot{V}_{12 b}=\sum_{i=1}^{N} \sum_{j \in \mathcal{N}_{i}}\left(\Delta_{i j}^{T} M \Delta_{i j}\right)
\end{aligned}
$$

Both terms are upper-bounded in what follows.

Upper-bound for $\dot{V}_{12 a}: \quad$ Using $x y \leq \frac{1}{2} x^{T} x+\frac{1}{2} y^{T} y, \dot{V}_{12 a}$ can be upper bounding

$$
\dot{V}_{12 a} \leq \sum_{i=1}^{N} \sum_{j \in \mathcal{N}_{i}} N_{i}\left(y_{i}^{j}-v_{i}^{j}\right)^{T} M\left(y_{i}^{j}-v_{i}^{j}\right)
$$


As the communication graph is undirected, one gets

$$
\dot{V}_{12 a} \leq \sum_{i=1}^{N} \sum_{j \in \mathcal{N}_{i}} N_{j}\left(y_{j}^{i}-v_{j}^{i}\right)^{T} M\left(y_{j}^{i}-v_{j}^{i}\right) .
$$

Upper-bound for $\dot{V}_{12 b}$ : Introducing $v_{i}^{j}$ in $\dot{V}_{12 b}$,

$$
\begin{aligned}
\dot{V}_{12 b}= & \sum_{i=1}^{N}\left(\sum_{j \in \mathcal{N}_{i}}\left(y_{i}^{j}-v_{i}^{j}\right)^{T} M\left(y_{i}^{j}-v_{i}^{j}\right)\right. \\
& +\sum_{j \in \mathcal{N}_{i}}\left(v_{i}^{j}-y_{i}^{i}\right)^{T} M\left(v_{i}^{j}-y_{i}^{i}\right) \\
& \left.+2 \sum_{j \in \mathcal{N}_{i}}\left(y_{i}^{j}-v_{i}^{j}\right)^{T} M\left(v_{i}^{j}-y_{i}^{i}\right)\right)
\end{aligned}
$$

Using again $x y \leq \frac{1}{2} x^{T} x+\frac{1}{2} y^{T} y$ one has

$$
\begin{aligned}
& 2 \sum_{j \in \mathcal{N}_{i}}\left(y_{i}^{j}-v_{i}^{j}\right)^{T} M\left(v_{i}^{j}-y_{i}^{i}\right) \\
\leq & \sum_{j \in \mathcal{N}_{i}}\left(y_{j}^{i}-v_{j}^{i}\right)^{T} M\left(y_{j}^{i}-v_{j}^{i}\right) \\
& +\sum_{j \in \mathcal{N}_{i}}\left(v_{i}^{j}-y_{i}^{i}\right)^{T} M\left(v_{i}^{j}-y_{i}^{i}\right)
\end{aligned}
$$

Injecting this expression in (59) leads to

$$
\begin{aligned}
\dot{V}_{12 b} \leq & \sum_{i=1}^{N}\left(2 \sum_{j \in \mathcal{N}_{i}}\left(y_{i}^{j}-v_{i}^{j}\right)^{T} M\left(y_{i}^{j}-v_{i}^{j}\right)\right. \\
& \left.+2 \sum_{j \in \mathcal{N}_{i}}\left(v_{i}^{j}-y_{i}^{i}\right)^{T} M\left(v_{i}^{j}-y_{i}^{i}\right)\right)
\end{aligned}
$$

As the communication graph is undirected, one gets

$$
\begin{aligned}
\dot{V}_{12 b} \leq & \sum_{i=1}^{N}\left(2 \sum_{j \in \mathcal{N}_{i}}\left(y_{j}^{i}-v_{j}^{i}\right)^{T} M\left(y_{j}^{i}-v_{j}^{i}\right)\right. \\
& \left.+2 \sum_{j \in \mathcal{N}_{i}}\left(v_{i}^{j}-y_{i}^{i}\right)^{T} M\left(v_{i}^{j}-y_{i}^{i}\right)\right)
\end{aligned}
$$


Upper bound for $\dot{V}_{12}$ : Finally, combining (58) and (60) in (56), one obtains

$$
\begin{aligned}
\dot{V}_{12} \leq & c_{1} \sum_{i=1}^{N}\left[\frac{1}{2 b_{i 2}}\left(z_{i}-N_{i} e_{i}^{i}\right)^{T} M\left(z_{i}-N_{i} e_{i}^{i}\right)\right. \\
& +\frac{b_{i 2}}{2} \sum_{j \in \mathcal{N}_{i}} N_{j}\left(y_{j}^{i}-v_{j}^{i}\right)^{T} M\left(y_{j}^{i}-v_{j}^{i}\right) \\
& +\left(z_{i}-N_{i} e_{i}^{i}\right)^{T} M \sum_{j \in \mathcal{N}_{i}}\left(v_{i}^{j}-y_{i}^{i}\right)+\frac{N_{i}}{2 b_{i}} e_{i}^{i T} M e_{i}^{i} \\
& +2\left(1+\frac{b_{i}}{2}\right) N_{i} \sum_{j \in \mathcal{N}_{i}}\left[\left(v_{i}^{j}-y_{i}^{i}\right)^{T} M\left(v_{i}^{j}-y_{i}^{i}\right)\right. \\
& \left.\left.+\left(y_{j}^{i}-v_{j}^{i}\right)^{T} M\left(y_{j}^{i}-v_{j}^{i}\right)\right]\right] .
\end{aligned}
$$

The upper bound for $\dot{V}_{1}$ becomes

$$
\dot{V}_{1} \leq x^{T} \bar{L} x+\sum_{i=1}^{N}\left(\tilde{\delta}_{i}-\rho z_{i}^{T} \Theta_{i} z_{i}\right)
$$

with $\tilde{\delta}_{i}, \rho$ and $\Theta_{i}$ as expressed in Theorem 2 .

As a consequence, if, for $i, j=1 \ldots N$, the communications are triggered when $\tilde{\delta}_{i} \geq \rho z_{i}^{T} \Theta z_{i}$, then $\dot{V}_{1} \leq 0$. The rest of the proof is identical to the one of Theorem 2.

\section{A.3. Proof of absence of Zeno behavior}

Two cases are considered: $D_{\max }=0$ and $D_{\max }>0$. Consider first the case with no perturbation $\left(D_{\max }=0\right)$. In this case, the estimate error $e_{i}^{i}$ vanishes. Moreover, since the initial states are assumed to be known by all agents, $y^{i}(t)=y^{j}(t)=v^{i}(t)$ for all $(i, j)$ and for all $t>0$. As a consequence, the discrepancies $y_{j}^{i}-v_{j}^{i}=0$ and $v_{i}^{j}-y_{i}^{i}=0$ for all $(i, j)$ and for all $t>0$. No communication will be triggered, which excludes the possibility of a Zeno behavior. Consider now the case with $D_{\max }>0$ and let us proove the absence of Zeno behavior. To do so, let us show that the inter-event time $t_{i, k+1}-t_{i, k}$ is strictly positive.

As the CTC (28) mainly depends on $e_{i}^{i}$, we begin by studying the time derivative of this error. From the definition of $e_{i}^{i}$ and by remarking that $u_{i}^{i}(t)=\tilde{u}_{i}^{i}(t)$, it can be expressed as

$$
\begin{aligned}
\dot{e}_{i}^{i} & =\dot{y}_{i}^{i}-\dot{x}_{i} \\
& =\left(A y_{i}^{i}+B \tilde{u}_{i}^{i}(t)\right)-\left(A x_{i}^{i}+B u_{i}^{i}+d_{i}\right) \\
& =A e_{i}^{i}-d_{i} .
\end{aligned}
$$

Then, it can observed that the derivative of $\left\|e_{i}^{i}\right\|$ satisfies

$$
\begin{aligned}
\frac{d}{d t}\left\|e_{i}^{i}\right\| & =\frac{e_{i}^{i T} \dot{e}_{i}^{i}}{\left\|e_{i}^{i}\right\|} \\
\frac{d}{d t}\left\|e_{i}^{i}\right\| & =\frac{1}{\left\|e_{i}^{i}\right\|} e_{i}^{i T}\left(A e_{i}^{i}-d_{i}\right) \\
\frac{d}{d t}\left\|e_{i}^{i}\right\| & \leq \frac{1}{\left\|e_{i}^{i}\right\|}\left(\|A\|\left\|e_{i}^{i}\right\|^{2}+\left\|e_{i}^{i}\right\| D_{\max }\right) \\
\frac{d}{d t}\left\|e_{i}^{i}\right\| & \leq\|A\|\left\|e_{i}^{i}\right\|+D_{\max },
\end{aligned}
$$


Solving the differential equation (63) leads to

$$
\left\|e_{i}^{i}\right\| \leq e^{\|A\|\left(t-t_{i, k}\right)} \alpha-\frac{D_{\max }}{\|A\|}
$$

for $t \geq t_{i, k}$ with $\alpha$ a constant. Remind that the error $e_{i}^{i}$ is reset to zero when a message is broadcast by Agent $i$, so $\left\|e_{i}^{i}\left(t_{i, k}\right)\right\|=0$. This is used to identify the value of $\alpha=\frac{D_{\max }}{\|A\|}$, and to obtain then the general solution of (63) for $t \geq t_{i, k}$ :

$$
\left\|e_{i}^{i}\right\| \leq\left(e^{\|A\|\left(t-t_{i, k}\right)}-1\right) \frac{D_{\max }}{\|A\|}
$$

From the CTC (28) a new communication will be triggered when $\tilde{\delta}_{i}=\rho z_{i}^{T} \Theta z_{i}+\eta$. Introducing

$$
\check{\delta}_{i}=\tilde{\delta}_{i}-e_{i}^{i T} M e_{i}^{i} \bar{b}_{i}
$$

a new communication is hence triggrered when

$$
\check{\delta}_{i}+e_{i}^{i T} M e_{i}^{i} \bar{b}_{i}=\rho z_{i}^{T} \Theta z_{i}+\eta
$$

and one has

$$
\begin{aligned}
\check{\delta}_{i}+\lambda_{\max }(M)\left\|e_{i}^{i}\right\|^{2} \bar{b}_{i} & \geq \rho z_{i}^{T} \Theta z_{i}+\eta \\
\left\|e_{i}^{i}\right\|^{2} & \geq \frac{1}{\lambda_{\max }(M) \bar{b}_{i}}\left(\rho z_{i}^{T} \Theta z_{i}-\check{\delta}_{i}+\eta\right)
\end{aligned}
$$

since $\lambda_{\max }(M)>0$ and $\bar{b}_{i}>0$. Using (68) along with (65) evaluated at time $t_{i, k+1}$, where the CTC is satisfied, allows to obtain

$$
\left(e^{\|A\|\left(t_{i, k+1}-t_{i, k}\right)}-1\right)^{2}\left(\frac{D_{\max }}{\|A\|}\right)^{2} \geq \frac{1}{\lambda_{\max }(M) \bar{b}_{i}}\left(\rho z_{i}^{T} \Theta z_{i}-\check{\delta}_{i}+\eta\right) .
$$

Considering the following two assumptions.

Assumption 1: $t_{i, k+1}=t_{i, k} \quad$ According to (16), (22), and (21), $\tilde{\delta}_{i}=0$ at $t=t_{i, k}$. As a consequence, the CTC (28) in Theorem 2 cannot be satisfied, which contradicts the considered assumption.

Assumption 2: $t_{i, k+1}>t_{i, k} \quad$ According to (62), for all $\left.t \in\right] t_{i, k}, t_{i, k+1}\left[\right.$ one has $\left\|e_{i}^{i}(t)\right\|>0$. Since $e_{i}^{i T} M e_{i}^{i} \geq$ $\left\|e_{i}^{i}\right\| \lambda_{\min }(M)$ and using the fact that $\lambda_{\min }(M)>0$ since $M=P B B^{T} P$ is symmetric positive, one deduces that $e_{i}^{i T} M e_{i}^{i}>0$ for all $\left.t \in\right] t_{i, k}, t_{i, k+1}\left[\right.$. This expression and (66) imply $\check{\delta}_{i}<\tilde{\delta}_{i}$ and

$$
\left(\rho z_{i}^{T} \Theta z_{i}-\check{\delta}_{i}+\eta\right)>0
$$

Then using (70) and $\left(e^{\|A\|\left(t_{i, k+1}-t_{i, k}\right)}-1\right)>0$ in (69), one gets

$$
\left(e^{\|A\|\left(t_{i, k+1}-t_{i, k}\right)}-1\right) \frac{D_{\max }}{\|A\|} \geq \sqrt{\frac{1}{\lambda_{\max }(M) \bar{b}_{i}}\left(\rho z_{i}^{T} \Theta z_{i}-\check{\delta}_{i}+\eta\right)} .
$$


In presence of perturbations, $D_{\max }>0$, thus

$$
\begin{aligned}
e^{\|A\|\left(t_{i, k+1}-t_{i, k}\right)} & \geq 1+\sqrt{\frac{\|A\|^{2}}{\lambda_{\max }(M) \bar{b}_{i} D_{\max }^{2}}\left(\rho z_{i}^{T} \Theta z_{i}-\check{\delta}_{i}+\eta\right)} \\
t_{i, k+1}-t_{i, k} & \geq \frac{1}{\|A\|} \ln \left[1+\sqrt{\frac{\|A\|^{2}}{\lambda_{\max }(M) \bar{b}_{i} D_{\max }^{2}}\left(\rho z_{i}^{T} \Theta z_{i}-\check{\delta}_{i}+\eta\right)}\right] .
\end{aligned}
$$

Since $\left(\rho z_{i}^{T} \Theta z_{i}-\check{\delta}_{i}+\eta\right)>0$, from (71), one deduces that $t_{i, k+1}-t_{i, k}>0$, which excludes the possibility of a Zeno behavior. 